Draft Version OCtober 29, 2018

Preprint typeset using $\mathrm{L}_{\mathrm{T}} \mathrm{EX}$ style emulateapj v. 01/23/15

\title{
THE PANCHROMATIC HUBBLE ANDROMEDA TREASURY. XVII. EXAMINING OBSCURED STAR FORMATION WITH SYNTHETIC ULTRAVIOLET FLUX MAPS IN M31.*
}

\author{
Alexia R. Lewis ${ }^{1,2,3}$, Jacob E. Simones ${ }^{4}$, Benjamin D. Johnson ${ }^{5}$, Julianne J. Dalcanton ${ }^{1}$, Evan D. Skillman ${ }^{4}$, \\ Daniel R. Weisz ${ }^{6}$, Andrew E. Dolphin ${ }^{7}$, Benjamin F. Williams ${ }^{1}$, Eric F. Bell ${ }^{8}$, Morgan Fouesneau ${ }^{9}$, Maria \\ Kapala ${ }^{10}$, Philip Rosenfield ${ }^{5}$, Andreas Schruba ${ }^{11}$ \\ Draft version October 29, 2018
}

\begin{abstract}
We present synthetic far- and near-ultraviolet (FUV and NUV) maps of M31, both with and without dust reddening. These maps were constructed from spatially-resolved star formation histories (SFHs) derived from optical Hubble Space Telescope imaging of resolved stars, taken as part of the Panchromatic Hubble Andromeda Treasury (PHAT) program. We use stellar population synthesis modeling to generate synthetic UV maps with a spatial resolution of $\sim 100$ pc ( $\sim 24$ arcseconds), projected. When reddening is included, these maps reproduce all of the main morphological features in the GALEX imaging, including rings and large star-forming complexes. The predicted UV flux also agrees well with the observed flux, with median ratios between the modeled and observed flux of $\log _{10}\left(f_{\mathrm{FUV}}^{\mathrm{syn}} / f_{\mathrm{FUV}}^{\text {obs }}\right)=0.03 \pm 0.24$ and $\log _{10}\left(f_{\mathrm{NUV}}^{\mathrm{syn}} / f_{\mathrm{NUV}}^{\mathrm{obs}}\right)=-0.03 \pm 0.16$ in the FUV and NUV, respectively. This agreement is particularly impressive given that we used only optical photometry to construct these UV maps. Having verified the synthetic reddened maps, we use the dust-free maps to examine properties of obscured flux and star formation. We compare our dust-free and reddened maps of FUV flux with the observed GALEX FUV flux and FUV $+24 \mu \mathrm{m}$ flux to examine the fraction of obscured flux. We find that the maps of synthetic flux require that $\sim 90 \%$ of the FUV flux in M31 is obscured by dust, while the GALEX-based methods suggest that $\sim 70 \%$ of the FUV flux is absorbed by dust. This $30 \%$ increase in the estimate of the obscured flux is driven by significant differences between the dust-free synthetic FUV flux and that derived when correcting the observed FUV flux for dust absorption with $24 \mu \mathrm{m}$ emission observations. The difference is further illustrated when we compare the SFRs derived from the FUV $+24 \mu \mathrm{m}$ flux with the $100 \mathrm{Myr}$ average SFR from the CMD-based SFHs. We find that the $24 \mu \mathrm{m}$-corrected FUV flux underestimates the SFR by a factor of $2.3-2.5$, depending on the chosen calibration. This discrepancy could be reduced by allowing for variability in the weight applied to the $24 \mu \mathrm{m}$ data, as has been recently suggested in the literature.

Keywords: galaxies: evolution - galaxies: individual (M31) - galaxies: star formation - galaxies: stellar content
\end{abstract}

\section{INTRODUCTION}

The star formation rate (SFR) of a galaxy is an extremely import astrophysical quantity, a key component to understanding the detailed evolution of a single galaxy

\footnotetext{
* Based on observations made with the NASA/ESA Hubble Space Telescope, obtained at the Space Telescope Science Institute, which is operated by the Association of Universities for Research in Astronomy, Inc., under NASA contract NAS 5-26555.

These observations are associated with program \#12055.

${ }^{1}$ Department of Astronomy, Box 351580, University of Washington, Seattle, WA 98195, USA

${ }^{2}$ Center for Cosmology and AstroParticle Physics, The Ohio State University, Columbus, OH 43210, USA

${ }^{3}$ Department of Astronomy, The Ohio State University, 140 West 18th Avenue, Columbus, OH 43210, USA

${ }^{4}$ Minnesota Institute for Astrophysics, University of Minnesota, 116 Church Street SE, Minneapolis, MN 55455, USA

${ }^{5}$ Harvard-Smithsonian Center for Astrophysics, 60 Garden Street, Cambridge, MA 02138, USA

${ }^{6}$ Department of Astronomy, University of California Berkeley, Berkeley, CA 94720, USA

${ }^{7}$ Raytheon, 1151 E. Hermans Road, Tucson, AZ 85756, USA

${ }^{8}$ Department of Astronomy, University of Michigan, 1085 S. University Ave., Ann Arbor, MI 48109, USA

${ }_{9}^{9}$ Max-Planck-Institut für Astronomie, Königstul 17, D-69117 Heidelberg, Germany

${ }^{10}$ Department of Astronomy, University of Cape Town, Private Bag X3, Rondebosch 7701, South Africa

${ }^{11}$ Max-Planck-Institut für extraterrestrische Physik, Giessenbachstrasse 1, 85748 Garching, Germany
}

or that of a population of galaxies across cosmic time. To this end, the ultraviolet (UV) is one of the most diagnostically important parts of a galaxy's spectrum. It is often interpreted as a measure of recent star formation, given that stars younger than $300 \mathrm{Myr}$, including young, massive $\mathrm{O}$ and $\mathrm{B}$ stars, emit most of their energy in the UV (e.g., Kennicutt \& Evans 2012). Additionally, the $\mathrm{UV}$ is essential for tracing star formation across cosmic time. At high redshift, the light observed in the optical is the galaxy's rest frame UV, which has been redshifted to longer wavelengths.

The UV, however, is also one of the most challenging parts of the spectrum to interpret reliably. While it is generally a good measure of recent star formation, the UV is also highly sensitive to dust. UV light is absorbed by dust grains, which re-emit in the infrared. Consequently, UV-only star formation rates (SFRs) underestimate the true SFR of a galaxy, usually by factors of a few in typical disk galaxies (e.g., Kennicutt 1998; Leroy et al. 2012), though it can be as much as a factor of 100 or more in ULIRGS (e.g., Schmitt et al. 2006). Additionally, stellar models in the UV are difficult to create and are neither well tested nor well-calibrated (e.g., Pradhan et al. 2014). Models are particularly problematic for highly evolved low-mass stars, which also emit modestly in the UV (e.g., Code 1969; O'Connell et al. 1992; 
Dorman et al. 1993; Rosenfield et al. 2012; Johnson et al. 2013), making the UV flux from older stellar populations particularly difficult to interpret (Conroy 2013). Finally, UV observations are difficult for low-redshift galaxies because they requires a space or balloon-borne mission, like the Galaxy Evolution Explorer (GALEX; Martin et al. 2005), the UVOT camera on Swift (Gehrels et al. 2004), or the UVIS channel on the Wide Field Planetary Camera 3 (WFC3/UVIS) aboard the Hubble Space Telescope.

Further complications when interpreting UV observations arise from assumptions on the initial mass function (IMF) and the SFR. Models generally assume that (1) the IMF is fully populated and (2) that the SFR is constant at recent times. The first of these assumptions is likely to hold only over large areas or in small but high star formation surface density regions. The second requirement arise because, at UV wavelengths, up to $90 \%$ of the emission in the far UV (FUV) and near UV (NUV) is from stars that are younger than $100 \mathrm{Myr}$ and $300 \mathrm{Myr}$, respectively; conversion to a SFR therefore assumes that the SFR has been uniform over that period of time (e.g., Kennicutt \& Evans 2012).

Although a wide range of science is enabled with these assumptions, the circumstances under which they are reliable remain unclear. For example, recent work on the IMF has shown that for sufficiently low SFRs and/or small spatial scales, the high-mass IMF is not fully populated (da Silva et al. 2012, 2014; Krumholz et al. 2015), and may even systematically vary (e.g., Meurer et al. 2009). Similarly, in low-mass galaxies and/or on small spatial scales in large galaxies, star formation histories (SFHs) tend to be bursty as opposed to constant (e.g., Lee et al. 2009; Weisz et al. 2012). The consequences of these deviations from the fiducial assumptions are not well-understood and may have a significant impact on our interpretation of the observed flux and consequently on the SFR.

Given the astrophysical importance of SFR measurements, tests of the above models and assumptions are crucial. A number of studies have examined how interpreting the observed galaxy flux is affected by some of these assumptions (e.g., Lee et al. 2009; Chomiuk \& Povich 2011; Weisz et al. 2012; Wilkins et al. 2012; Johnson et al. 2013; Boquien et al. 2014; Simones et al. 2014, among many others). In a recent paper, Boquien et al. (2015) analyzed the scale dependence of SFR tracers. They found broad agreement between tracers on $\sim 1 \mathrm{kpc}$ scales, presumably because variations between regions of active star formation and diffuse emission (i.e., from older stellar populations) have averaged out. The scale at which this occurs will vary from galaxy to galaxy depending on the intensity of star formation as well as the structure and transparency of the interstellar medium.

In this paper, we take an alternate approach to SFR analysis that sidesteps some of the above assumptions. We use SFHs and dust distributions derived from resolved stars and stellar population synthesis models to create maps of synthetic FUV and NUV flux on subkpc scales. The SFHs come from Lewis et al. (2015), who used optical ${ }^{13}$ resolved star data, taken as part of

13 Although PHAT includes data in two UV filters (F275W and F336W), that data is not used in this analysis. Only the brightest main sequence stars have measurements in the UV filters, severely

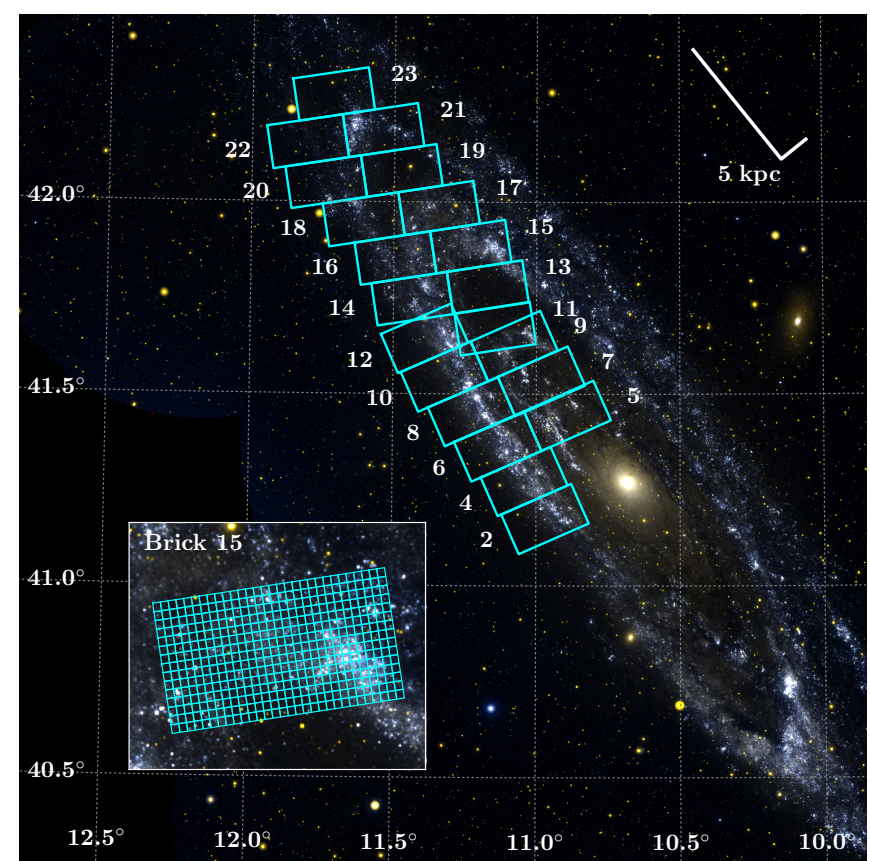

Figure 1. Map of the PHAT survey area. The 21 PHAT bricks analyzed in this study are outlined and numbered. Each brick was divided into 450 regions on a $15 \times 30$ grid, as shown for Brick 15 in the inset panel. The SFHs for each region are presented in Lewis et al. (2015). We note that Bricks 1 and 3 (nearest to the center of the galaxy) are not used in the present study because their CMDs are too shallow for reliable SFH determination. In the upper right image we include a scale bar denoting $5 \mathrm{kpc}$ along the major and minor axes of the galaxy. The image is oriented such that north is up and east is to the left.

the Panchromatic Hubble Andromeda Treasury (PHAT; Dalcanton et al. 2012; Williams et al. 2014) program, to model the spatially-resolved recent $\mathrm{SFH}$ of $\sim 9000$ regions ( $\sim 0.01 \mathrm{sq} . \mathrm{kpc}$ in size) in the northeast quadrant of M31 (Figure 1). Use of these detailed SFHs allows us to relax the constant SFR assumption, and the poisson likelihood sampling in the $\mathrm{SFH}$ derivation process implicitly corrects the SFHs for IMF sampling, which is included in the uncertainty estimate. We compare the synthetic flux maps with far- and near-UV GALEX observations (Morrissey et al. 2007) to analyze the effect of non-constant SFHs on UV flux over a wide range of environments within a single galaxy. This methodology facilitates an end-to-end test verifying the connection between massive star formation, UV flux, and the ability to interpret and/or infer UV populations through analysis of optical stellar populations.

Simones et al. (2014) initiated this work in M31, using techniques similar to that of Johnson et al. (2013) to model the UV flux in 33 star-forming regions in M31's star-forming ring and compare UV flux-derived SFRs to those measured from optical color-magnitude diagram (CMD)-derived SFHs. They found that the SFRs derived from CMDs were, on average, consistent with those derived from the extinction-corrected FUV flux to within $1 \sigma$, and that $\sim 1 / 3$ of the scatter could be attributed to metallicity differences: the flux calibration assumes constant solar metallicity, while the metallicity of the SFHs

limiting the age range over which the SFH would be reliable. The optical data are the deepest and therefore provide the best leverage on measuring the SFH. 
varies with time. Additionally, Simones et al. (2014) found that a wide range of SFHs can produce the same amount of FUV flux.

The analysis of resolved stellar populations offers a different way to probe the effects of simplifying assumptions on integrated flux measurements. Unfortunately, tests such as the one performed by Simones et al. (2014) have been limited in scope, both due to the inability to gather the required resolved star data outside of the Local Group and because the vast majority of galaxies in the nearby universe are dwarf galaxies. Johnson et al. (2013) explored the connection between the UV and resolved stellar populations in nearby low-mass, low-metallicity dwarf galaxies using SFHs from the ACS Nearby Galaxy Treasury program (ANGST; Dalcanton et al. 2009; Weisz et al. 2011). They found that fluctuations in the SFH can cause factor of two variations in UV luminosities relative to constant SFR assumptions, and that stars older than $100 \mathrm{Myr}$ can contribute up to $30 \%$ of FUV emission.

Using the SFHs from resolved CMD fitting is just one way to model the UV flux with stellar photometry. Another method is to fit the spectral energy distributions (SEDs) of individual stars (e.g., Romaniello et al. 2002; Maíz-Apellániz 2004; Robitaille et al. 2007; Bailer-Jones 2011; Bianchi et al. 2012a,b). SED fitting is often performed to recover the properties of the star and the surrounding interstellar medium $\left(T_{\mathrm{eff}}, \log g, Z, A_{V}\right)$. Such fitting, though, ideally produces the star's full spectrum, both reddened and intrinsic, which can then be used to infer the flux at wavelengths that were not observed. The PHAT survey motivated the development of the Bayesian Extinction and Stellar Tool (BEAST; Gordon et al. 2016), an SED fitter, which could be used to provide and independent check of the results presented in this paper. SED fitting will, however, miss any stars below the detection limit because it requires that they be detected in multiple filters. In contrast, the CMD modeling used in this paper includes the UV flux for the entire stellar population.

Making this measurement in a large, Milky Way-like galaxy is important for interpreting the observations of massive galaxies that dominate star-forming galaxy samples. In the nearby universe, we are limited to only a few large spiral galaxies with sufficiently well-resolved populations. Of these, M31 data has the widest wavelength coverage, the best resolved star data, and the highest resolution. M31 is a more massive and metal-rich system than nearby dwarf galaxies, providing an important laboratory for testing the assumptions made when using integrated UV light. While the method for studying UV populations is the same as that in lower-mass systems, M31's environment is critical because it is similar to that in which most stars are formed.

This paper is organized as follows. In Section 2, we describe the SFH dataset and the GALEX FUV and NUV images used, and the production of the synthetic flux maps. Section 3 describes the creation of the UV maps, including the methodology used to model the UV fluxes and the manner in which we assembled the maps. We present the maps in Section 4 and discuss factors that affect the synthetic maps, including a discussion of uncertainties in the modeling. In Section 5, we examine results from this analysis, including the fraction of obscured star formation and comparison of SFR calibrations. We discuss the factors that affect our results in Section 6. We conclude in Section 7.

\section{DATA}

\subsection{GALEX UV Images}

We took UV observational data from the GALEX Deep Imaging Survey (DIS; Martin et al. 2005), using the five tiles that cover the PHAT survey in the FUV and NUV. These tiles are listed in Table 1. We masked the tile edges and converted the count rate units, cps, into flux according to

$$
f=U\left(\frac{\mathrm{cps}}{\text { counts s}^{-1} \text { pixel }^{-1}}\right),
$$

where $U$, the GALEX unit response, is given in Table 2. A small amount of background UV flux was present in the FUV and NUV mosaics, primarily due to scattering of UV photons from hot foreground stars in the Galaxy. We estimated the background as follows: In each of the five tiles, we measured the mean flux in four $0.06 \times 0.06$ square degree apertures in off-galaxy areas relatively devoid of stars. The centers of each of the twenty apertures are listed in Table 1 next to the appropriate tile. We then averaged these twenty measurements to get our estimate of the background flux and subtracted this value from all pixels in the corresponding mosaic. The subtracted background in the FUV and NUV images was $3.054 \pm 0.601 \times 10^{-16} \mathrm{erg}^{-1} \mathrm{~s}^{-1} \mathrm{~cm}^{-2} \AA^{-1}$ and $2.386 \pm 0.678 \times 10^{-16} \mathrm{erg} \mathrm{s}^{-1} \mathrm{~cm}^{-2} \AA^{-1}$, respectively.

\subsection{PHAT SFHs}

To model UV flux, we use the PHAT spatially-resolved recent SFHs determined in Lewis et al. (2015). We briefly describe their derivation here, but refer readers to the original paper for details. Each brick in the PHAT survey was divided into 450 regions on a uniform $15 \times 30$ grid with a total of $\sim 9000$ regions across the survey area, excluding the bricks closest to the crowded bulge area (see Figure 1). Each region was approximately $24^{\prime \prime} \times 27^{\prime \prime}(100$ pc $\times 100$ pc, projected; $100 \mathrm{pc} \times 400 \mathrm{pc}$, deprojected). For each region, they modeled the optical (F475W and F814W) CMD using the fitting code MATCH (Dolphin 2002), which compares the observed CMD with many synthetic CMDs for composite stellar populations over a range of ages and metallicities. As described in detail in Lewis et al. (2015), the SFHs were derived with the following assumptions:

\section{A Kroupa (2001) IMF.}

2. Padova isochrones (Marigo et al. 2008) with updated asymptotic giant branch (AGB) tracks (Girardi et al. 2010).

3. A distance modulus of 24.47 , corresponding to a distance of $783 \mathrm{kpc}$ (McConnachie et al. 2005).

4. A binary fraction of 0.35 with a uniform mass ratio distribution between 0 and 1 .

5. Age resolution of 0.1 dex for $\log$ (time) $=6.6-9.9$ and 0.25 dex for $\log ($ time $)=9.9-10.15$ (In Section 3.1 , we correct the youngest time bin so that it extends to the present day.) 
Table 1

GALEX Observations.

\begin{tabular}{cccccc}
\hline \hline Tilename & RA & dec & \multicolumn{2}{c}{ exposure time $(\mathrm{s})$} & Background Aperture Centers \\
& & & FUV & NUV & $(\mathrm{J} 2000)$ \\
\hline PS_M31_MOS00 & 10.675 & 41.267 & 9863.55 & 95842.35 & $(11.02,40.78),(11.25,41.02),(10.47,41.80),(9.94,41.39)$ \\
PS_M31_MOS07 & 10.600 & 42.350 & 7418.25 & 50119.15 & $(10.06,42.13),(10.82,42.21),(10.82,42.73),(9.92,42.48)$ \\
PS_M31_MOS08 & 11.350 & 42.200 & 7497.6 & 55819.3 & $(11.77,42.62),(12.02,42.03),(10.70,42.16),(10.93,42.63)$ \\
PS_M31_MOS09 & 12.170 & 42.032 & 5431.7 & 48263.9 & $(11.85,41.98),(12.38,42.21),(11.98,41.49),(12.48,42.53)$ \\
PS_M31_MOS10 & 11.220 & 41.370 & 6561.5 & 51551.05 & $(11.83,41.40),(11.76,41.64),(11.48,40.99),(11.07,41.04)$ \\
\hline
\end{tabular}

Note. - Tilename, RA, dec, exposure time in the FUV and NUV, and the centers of the four apertures in which the background was measured for each tile used to create the GALEX UV mosaics.

6. Metallicity resolution of 0.1 dex over the range $-2.3 \leq[\mathrm{M} / \mathrm{H}] \leq 0.1$, with the requirement that $[\mathrm{M} / \mathrm{H}]$ increases with time.

7. A two-parameter extinction model consisting of a foreground component, $A_{V}$, applied evenly to all stars, and an additional differential component, $d A_{V}$ applied to stars following a uniform distribution, such that all stars in a region are extincted by some amount between $A_{V}$ and $A_{V}+d A_{V}$, optimized for each region individually (see also Simones et al. 2014). These values are used in a Cardelli et al. (1989) extinction model with $R_{V}=3.1$.

Additionally, the portion of each CMD with F475W > 21 and $\mathrm{F} 475 \mathrm{~W}-\mathrm{F} 814 \mathrm{~W}>1.25$ (red giant branch and red clump stars) was excluded from the fit (see the CMDs in Figures 2 and 3 of Lewis et al. 2015). This choice mitigates extinction effects from older stellar populations which are not well fit with the single step function described in item 7 above. As a result, the optimized extinction parameters correspond only to the dust associated with young, UV-emitting stars on the main sequence. The SFHs are therefore limited to the last 500 Myr. We refer the reader to Dalcanton et al. (2015) for a robust analysis of the dust in M31. The full SFH analysis using the red features of the CMD and the Dalcanton et al. (2015) dust analysis is the subject of an upcoming paper (B. Williams et al., in prep.).

We also note that the SFHs of Lewis et al. (2015) do not differentiate between cluster and field stars. As a result, UV light from young clusters is included in this analysis. However, stellar clusters account for only $4-$ $8 \%$ of the star formation in M31 (Johnson et al. 2016), and they therefore contribute only a few percent of the integrated UV light.

\section{MAP CREATION}

\subsection{Modeling Ultraviolet Flux}

We used the SFHs described in Section 2.2 to create spatially-resolved broadband FUV and NUV flux maps for the PHAT survey area. We modeled the flux in each region using a technique similar to that described in Johnson et al. (2013). We generated a set of simple stellar population (SSP) models and then weighted those models by the SFH to calculate the integrated SED for a given region. We ultimately determined the FUV and NUV flux and magnitude of each region from its modeled SED.

We first constructed a set of SSPs using the Flexible Stellar Population Synthesis (FSPS) code (Conroy et al.
2009; Conroy \& Gunn 2010), assuming a Kroupa (2001) IMF and the Padova isochrones (Marigo et al. 2008) with updated AGB tracks (Girardi et al. 2010) and using the BaSeL 3.1 semi-empirical stellar SED library (Westera et al. 2002). In FSPS, the Geneva tracks (Meynet et al. 1994) are used at $\log$ (time) $<6.6$ for high-mass stars, supplemented with the Padova models for low mass stars. These choices are consistent with the SFH determination. We constructed the SSPs with an age resolution of 0.025 dex over the range $\log$ (age) from 5.500 to 10.175 . We set the SSP metallicity to the mean metallicity over the last $100 \mathrm{Myr}$, as derived from the SFH. If there was no star formation over that time range, we set the metallicity to that of the most recent time when the SFR was non-zero.

To link the SSPs to the SFH, we needed to reprocess the SFH. The MATCH implementation of the Padova isochrones only reaches to $\log ($ time $)=6.6$, so we renormalized the SFR in the youngest age bin to reach time $=0$, conserving the mass created in that time bin:

$$
\begin{aligned}
& \operatorname{SFR}\left(t=0-10^{6.7} \mathrm{Myr}\right)= \\
& \quad \operatorname{SFR}\left(t=10^{6.6}-10^{6.7} \mathrm{Myr}\right) \times\left(1.0-\frac{10^{6.6}}{10^{6.7}}\right) .
\end{aligned}
$$

We also increased the age resolution of the $\mathrm{SFH}$ to $\sim 6.5 \times 10^{4} \mathrm{yr}$. We determined this value by splitting the smallest time bin into 20 separate bins. In $\log$ (time) space we then interpolated the SSP SEDs to the SFH time points, weighted each interpolated SED by the SFR at each SFH time point, and summed the SEDs to create the integrated intrinsic (i.e., dust-free) model SED. We note that we used the full $14 \mathrm{Gyr} \mathrm{SFH}$ in this process despite the fact that Lewis et al. (2015) stress that the SFHs are only robust to $\sim 500 \mathrm{Myr}$ ago. We will discuss the effects of the SFH timescale in the Appendix.

To include the effects of dust, we created the integrated, attenuated model SED in the same manner as the intrinsic SED, except that we reddened each individual SSP SED component before weighting by the mass. To redden the SED, we split each SSP SED into 30 identical component SEDs. Each component was then attenuated according to the Cardelli et al. (1989) extinction curve with $R_{V}=3.1$, assuming a uniform random $A_{V}$ distribution drawn between $A_{\mathrm{V}, \mathrm{SFH}}$ and $A_{\mathrm{V}, \mathrm{SFH}}+d A_{\mathrm{V}, \mathrm{SFH}}$, where $A_{\mathrm{V}, \mathrm{SFH}}$ and $d A_{\mathrm{V}, \mathrm{SFH}}$ are the best-fit parameters of the two-component extinction model used to derive the SFH from the CMD (Lewis et al. 2015). This was done to mimic the SFH derivation, as described briefly in bullet point 7 of Section 2.2.

We summed all of the attenuated components to create 
Table 2

$G A L E X$ filter properties.

\begin{tabular}{lrc}
\hline \hline & FUV & NUV \\
\hline Unit response, $U\left(\times 10^{-15} \mathrm{erg} \mathrm{s}^{-1} \mathrm{~cm}^{-2} \AA^{-1}\right)^{\mathrm{a}}$ & 1.40 & 0.206 \\
AB magnitude zeropoint, $Z$ & 18.82 & 20.08 \\
\hline a $\quad$ http://asd.gsfc.nasa.gov/archive/galex/FAQ/counts \\
background.html
\end{tabular}

each region's integrated attenuated model SED. We note that the Cardelli et al. (1989) extinction curve, which predicts the amount of extinction relative to that in the $V$ band as a function of wavelength, is based on the average $R_{\mathrm{V}}=3.1$ extinction curve for the Galaxy. Previous studies have shown that this extinction curve is applicable to M31 as a whole in both the UV (Bianchi et al. 1996) and the optical (Barmby et al. 2000) regimes. However, individual sight-lines may differ from $R_{\mathrm{V}}=3.1$ due to metallicity, gas-to-dust ratio, or star formation activity (Clayton et al. 2015).

To determine the UV magnitude, we projected each model SED (both intrinsic and attenuated) onto the response curves for the GALEX FUV and NUV filters to obtain absolute synthetic FUV and NUV magnitudes in the $\mathrm{AB}$ system. We converted the resulting absolute magnitude to apparent magnitude assuming a distance modulus of 24.47 (McConnachie et al. 2005).

Finally, we convert the magnitude to flux using:

$$
m=-2.5 \log _{10}\left(\frac{f}{U}\right)+Z,
$$

where $U$ is the GALEX unit response, and $Z$ is the zeropoint, given for each filter in Table 2 .

Throughout the text, we refer to the synthetic intrinsic (un-reddened) flux as $f^{\text {syn, } 0}$ and the synthetic reddened flux as $f^{\text {syn }}$. The same nomenclature is used when referring to synthetic magnitudes as well.

We note that this process can be applied when modeling the flux at any wavelength, although appropriate care must be taken in the IR, where dust geometry and radiative transfer effects also need to be considered.

\subsection{Creating Maps of Synthetic Flux}

We used the modeled FUV and NUV fluxes in each region to create a single map of the PHAT survey area at each wavelength. We created these maps with Mon$\operatorname{tage}^{14}$, which aids in the combination of many FITS images into a single mosaic. Montage is flux-conserving and maintains the photometric and spatial fidelity of the input images.

Because the SFHs were derived on a $15 \times 30$ grid in each brick, we assembled the modeled flux values for each UV filter into a $15 \times 30$ array to create a brick image. We then tied each brick image independently to a world coordinate system using the RA and Dec coordinates of the SFH brick grid. We then let Montage determine the single template header that best describes the combination of input images and used that header to re-project each brick image to the same WCS. We co-added the re-projected images into a single FITS file to create full

14 http://montage.ipac.caltech.edu
PHAT survey area maps of synthetic FUV and NUV flux. The pixels in the resulting co-added image have a scale of $23.7^{\prime \prime}$. Due to the reprojection, the input regions do not match one-to-one to the output image pixels. We do not background-match the synthetic images.

The rotation of the outer bricks with respect to the inner bricks results in a number of locations where pixels in one brick overlap with pixels in another brick, most notably in Bricks 9 and 11. We note, however, that three of the fields in B11 were not observed. These three fields overlap completely with fields from B09, decreasing the total amount of overlap. We compared the output reddened NUV flux values in 10 of these overlapping pixels. We found the agreement to be within $10-15 \%$ on average.

Assuming a distance modulus of 24.47 (McConnachie et al. 2005), a disk inclination of $77^{\circ}$ (e.g., Roberts 1966; Brinks \& Burton 1984; Walterbos \& Kennicutt 1988), and a major axis position angle of $35^{\circ}$ (de Vaucouleurs et al. 1995), the pixel scale deprojects to a linear size of $440 \mathrm{pc} \times 100 \mathrm{pc}$ along the minor and major axes of M31, respectively (see orientation of scale bar in Figure 1). The synthetic flux maps therefore have a resolution that is firmly in the sub-kpc regime.

Montage creates a pixel weight map during the mosaicking process. All pixels are weighted according to the fraction of the pixel that is inside the PHAT footprint (i.e., pixels entirely inside the footprint have a weighting of 1 , pixels entirely outside of the PHAT footprint have a weighting of 0 , and pixels that are partially within the footprint have a weighting somewhere between 0 and 1 ). To remove possible border effects, we only analyze pixels that have a weight $w>0.95$.

\subsection{Turning GALEX Observations into Maps}

We also constructed maps of observed GALEX flux $\left(f^{\text {obs }}\right)$, matched to the sampling of the modeled synthetic flux maps. The process was similar to that used for the synthetic maps. Starting with the backgroundsubtracted images described in Section 2.1, we reprojected the flux tiles to the same template header as the synthetic flux maps. We then background-matched the images by determining the differences in the images at their overlaps and fitting a plane to these difference images to model the background. Finally, we co-added the matched images to create the final observed maps.

\section{VERIFICATION OF THE OPTICALLY-DERIVED SYNTHETIC ULTRAVIOLET MAPS OF THE PHAT SURVEY \\ 4.1. Presentation of the Maps}

In Figures 2 and 3 we show maps of observed GALEX flux $\left(f^{\text {obs }} ; \S 3.3\right)$, synthetic attenuated flux $\left(f^{\text {syn }} ; \S 3.1\right.$ and $\S 3.2$ ), and the significance of the difference between the two over the PHAT survey area for the FUV and NUV, respectively. All figures have the same stretch.

Figures 2 and 3 show remarkable qualitative agreement between the synthetic and the observed fluxes, indicating that the synthetic attenuated fluxes derived from the optical CMDs do an excellent job at reproducing the observed fluxes. All of the main features of the GALEX maps are reproduced in the synthetic maps, including the $10 \mathrm{kpc}$ ring, the ring features at 5 and $15 \mathrm{kpc}$, and the individual star-forming regions within the ring, as 


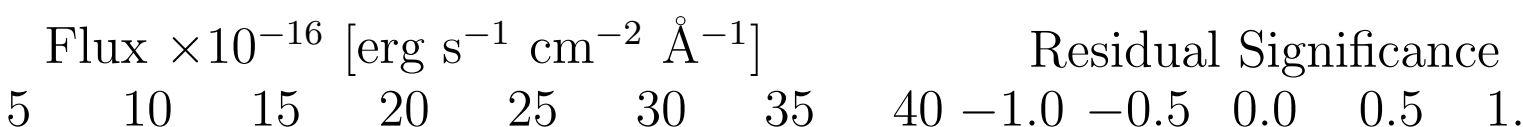

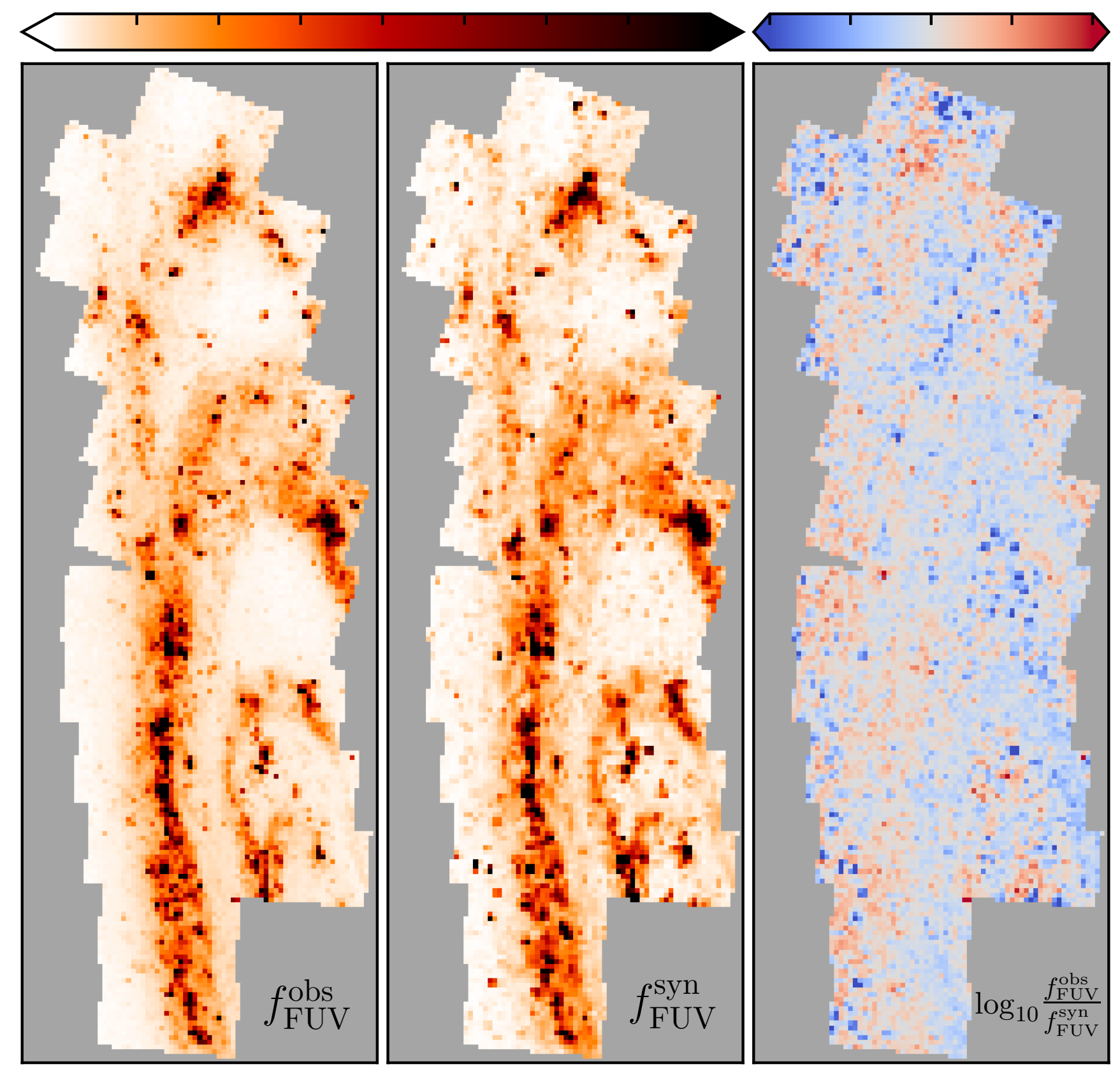

Figure 2. The observed GALEX FUV flux, $f_{\mathrm{FUV}}^{\text {obs }}$, is shown in the left panel. The middle panel shows the synthetic flux derived from optical SFHs and attenuated according to a Cardelli extinction model, $f_{\mathrm{FUV}}^{\mathrm{syn}}$. These maps are on the same flux scale as indicated by the color bar above the left two panels. The right panel shows the significance of the residuals, plotted as $\log \left(f^{\text {obs }} / f^{\text {syn }}\right)$. Bluer colors are where the modeled flux is over-predicted and redder colors are where it is under-predicted.

well as the OB associations in Bricks 15 and 21 (the bright features located at the top and right side of the left two panels in Figures 2 and 3; also see Figure 1 for brick numbering). The agreement is especially good in the FUV map, but also apparent in the NUV map, despite the fact that some features are not as defined in the synthetic map. We emphasize that we have used optical colors and magnitudes to derive the ultraviolet fluxes.

There are, however, distinct differences between the $f^{\text {syn }}$ and $f^{\text {obs }}$ maps. For example, the $f^{\text {obs }}$ maps show some point-like sources that do not appear in the $f^{\text {syn }}$ maps. Inspection of the original images shows that these sources are largely Milky Way foreground stars. Foreground stars are typically at a very different absolute magnitude than M31 stars, and it is unlikely that any foreground stars were inadvertently included in the CMD modeling. Additionally, foreground stars don't produce features in the synthetic maps because the fluxes in those 
Flux $\times 10^{-16}\left[\mathrm{erg} \mathrm{s}^{-1} \mathrm{~cm}^{-2} \AA^{-1}\right] \quad$ Residual Significance

$\begin{array}{lllllllllllll}5 & 10 & 15 & 20 & 25 & 30 & 35 & 40 & -1.0 & -0.5 & 0.0 & 0.5 & 1.0\end{array}$

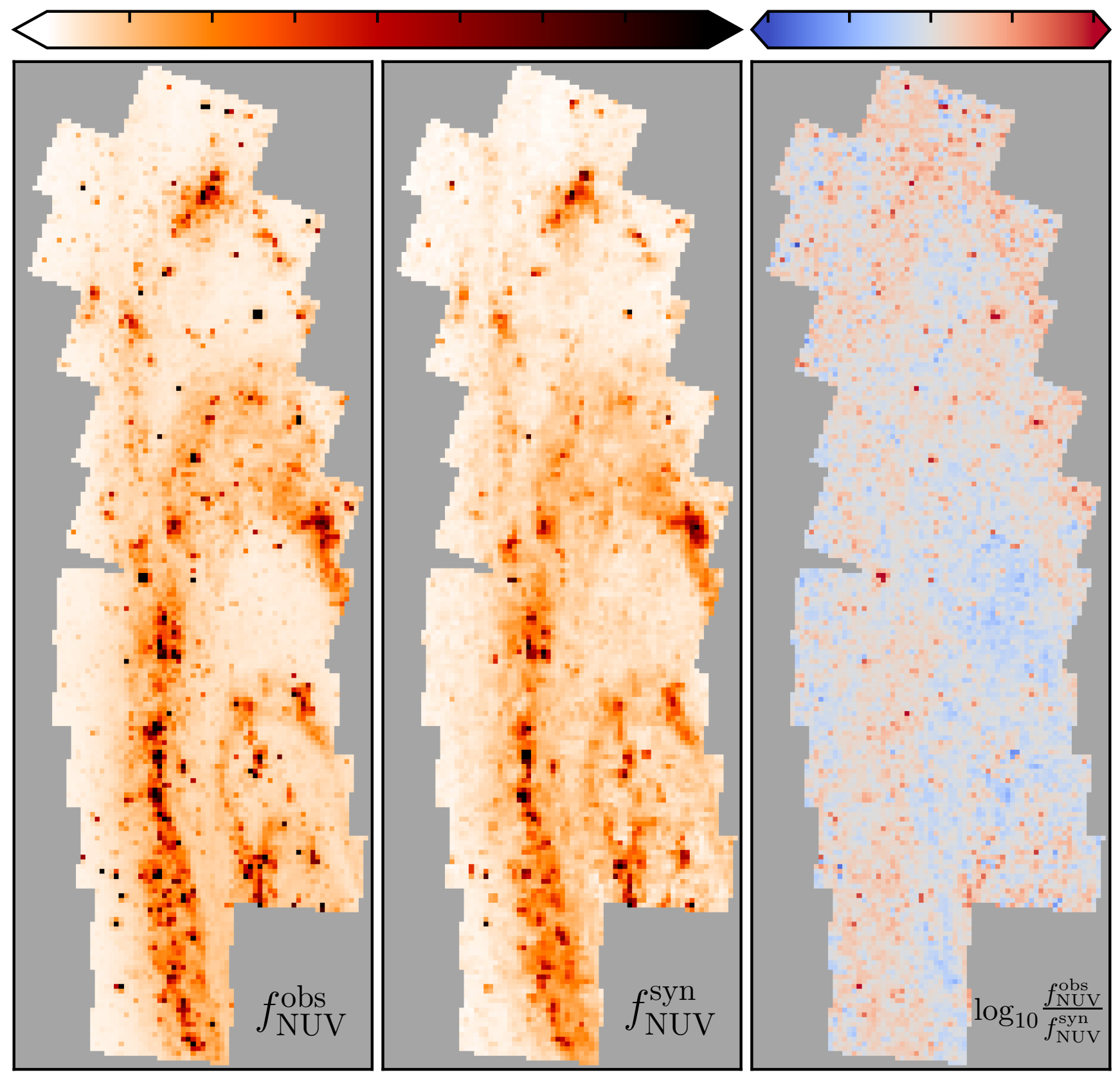

Figure 3. Same as Figure 2 except showing the NUV results. The left panel shows the observed flux, $f_{\mathrm{NUV}}^{\text {obs }}$, the middle panel shows the modeled, attenuated flux, $f_{\mathrm{NUV}}^{\mathrm{syn}}$, and the right panel shows the significance of the residuals between the two.

maps are derived from the SFR of a distribution of stars, rather than single stars, so while a single bright star may dominate the pixel in the observed map, it has less of an effect in the modeled map because it is averaged out in the total SFH from which the modeled flux is derived.

There are also a number of bright pixels in the modeled maps that are not in the observed maps. These mostly correspond to photometric artifacts (primarily diffraction spikes) that remained in the PHAT photometry ${ }^{15}$

15 We note that the SFHs derived in Lewis et al. (2015) used after application of the quality cuts (Dalcanton et al. 2012). These artifacts increase the apparent stellar mass measurement in the SFH, which increases the synthetic flux. This contamination affects $<5 \%$ of the pixels.

In Figure 4, we present a quantitative comparison

the first version of the PHAT photometry. The photometry has since changed, using different quality cuts and resulting in overall improvement. Given the large computing expense to run the SFHs on the entire grid and the minimal difference it would make for the SFHs, it was not deemed worthwhile to re-run the SFHs on the most recent photometry. 

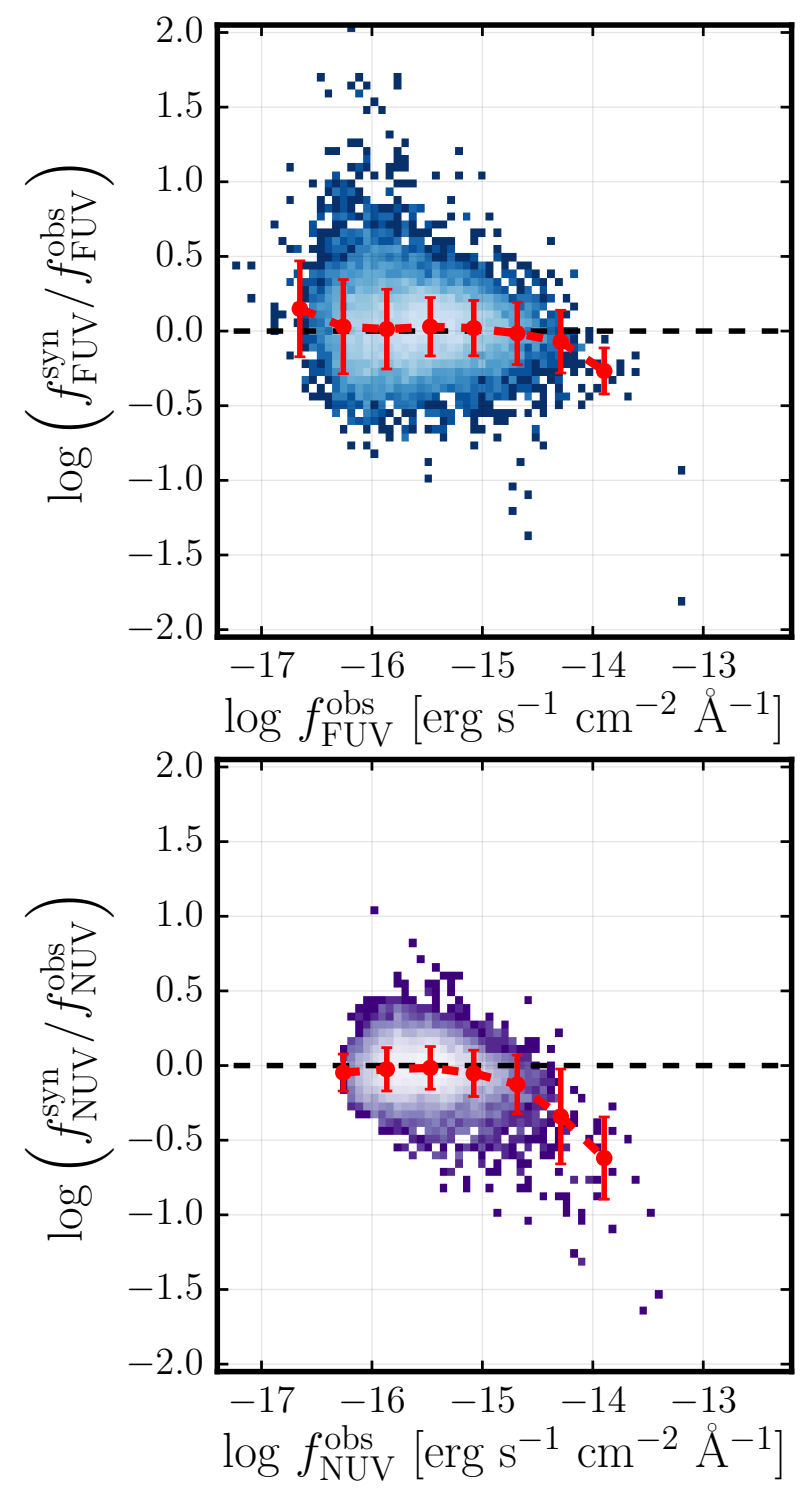

Figure 4. The log ratio of the modeled flux to the observed flux as a function of observed flux for all pixels that fall inside the PHAT footprint. The red circles in each panel show the running median with the standard deviation given by the error bars on each point. The median log ratios are 0.005 and -0.027 in the FUV and NUV, respectively. The standard deviation is 0.24 in the FUV and 0.16 in the NUV.

of the synthetic and observed fluxes shown in Figures 2 and 3. We plot the ratio of the modeled reddened flux to the GALEX flux $\left(f^{\text {syn }} / f^{\text {obs }}\right)$ as a function of GALEX flux, including the running median and standard deviation. The overall median and standard deviation are $\log _{10}\left(f_{\mathrm{FUV}}^{\mathrm{syn}} / f_{\mathrm{FUV}}^{\text {obs }}\right)=0.03 \pm 0.24$ and $\log _{10}\left(f_{\mathrm{NUV}}^{\mathrm{syn}} / f_{\mathrm{NUV}}^{\mathrm{obs}}\right)=-0.03 \pm 0.16$. Together with the qualitative comparison in Figures 2 and 3, these numbers indicate that in both filters, $f^{\text {syn }}$ is consistent with $f^{\text {obs }}$. The largest average discrepancy occurs at high observed flux, where the number of regions is very small, and are likely due to foreground stars or other photometric artifacts.

The FUV relation is flat across the range of observed fluxes. The scatter on the best-fit values decreases as the observed flux increases, ranging from 0.3 dex at the low end to 0.1 dex at the high end. The two regions at the highest FUV flux actually correspond to a single bright star located at the intersection of B12 and B14, clearly visible in Figure 1 and in the left panel of Figure 2. On a region-by-region basis, regions of low observed flux show the largest deviations between the observed and synthetic flux.

In contrast, the NUV data are much tighter, showing a scatter of $\sim 0.1$ dex at low observed fluxes and increasing to $\sim 0.2$ dex at the brighter end. There is also a slight trend in the NUV data. The synthetic flux is generally in very good agreement in regions of low observed flux but under-estimated in regions of high observed flux. This behavior can be seen in the right panel of Figure 3 where the regions between and outside of the ring features (low observed flux) are gray to light blue in color, indicating only slightly more synthetic flux than observed flux, and the ring regions (high observed flux) are red, indicating more observed flux than synthetic flux.

At the high flux end of the NUV distribution, the inconsistency must lie with the modeled data. One possibility could be our treatment of dust, because the regions of high flux coincide with the dustiest regions. However, both the FUV and NUV are treated the same way and this problem does not affect the FUV, which should be even more sensitive to errors in the dust model; we cannot assume that the dust is too high in the NUV and just right in the FUV. We note, however, that the NUV band includes the $2175 \AA$ bump. If we were to reduce the strength of the bump at the same $R_{V}$ (e.g., Conroy et al. 2010), this change would increase the flux in the NUV, bringing the ratio of synthetic to observed flux closer to one but leaving the FUV flux unchanged. A variation in bump strength would also have little effect on the low-flux regions which are also generally low-dust regions and are therefore less affected by the robustness of the extinction curve.

A final factor that could contribute to the trend seen in the NUV data is timescale. When we constructed the set of SSPs, we set the metallicity of each SSP to the mean metallicity of the SFH over the last 100 Myr. The 100 Myr timescale is based on the average lifetime of the $\mathrm{O}$ and B stars that emit in the FUV, whereas the timescale for NUV emission is longer at $300 \mathrm{Myr}$. We tested using $300 \mathrm{Myr}$ as the baseline over which to determine the mean metallicity for each SSP and found no difference in the results. M31's metallicity has not changed significantly over the last few hundred Myr. Therefore, metallicity is not the reason for the observed trend.

We convert flux to magnitude and compare maps of observed and synthetic UV color $\left(m_{\mathrm{FUV}}-m_{\mathrm{NUV}}\right)$ in Figure 5. The left panel shows the observed color, the middle panel shows the predicted, reddened color, and the right panel shows the difference between the two. The main difference between the left and middle panels is that the predicted map is bluer in the star-forming regions along the $10-\mathrm{kpc}$ ring. This is because the synthetic NUV flux is slightly under-predicted in the high flux, star-forming regions, resulting in fainter NUV magnitudes and therefore bluer colors. This is confirmed in the third panel, which shows the difference between the observed and predicted colors.

Despite the systematic trend in the NUV and the larger scatter in the FUV, we find that $70-75 \%$ of pixels in 
$m_{\mathrm{FUV}}-m_{\mathrm{NUV}}$

observed - predicted

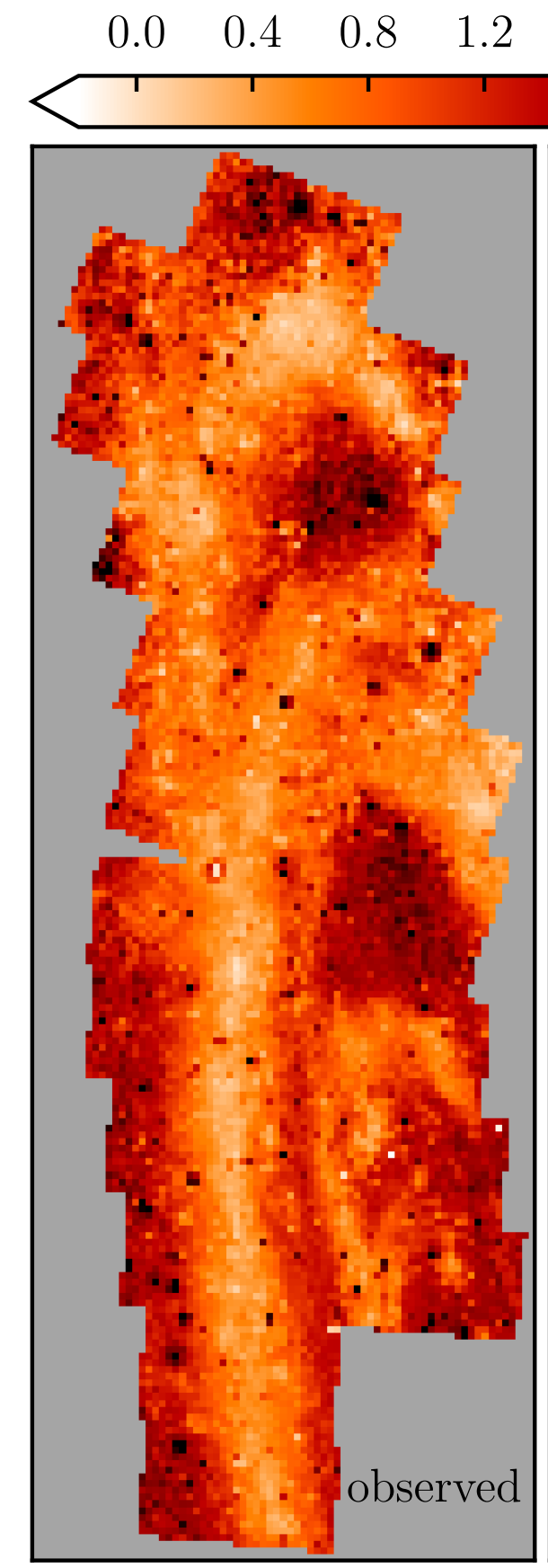

$1.6 \quad 2.0$

2.4

2.8

$-$
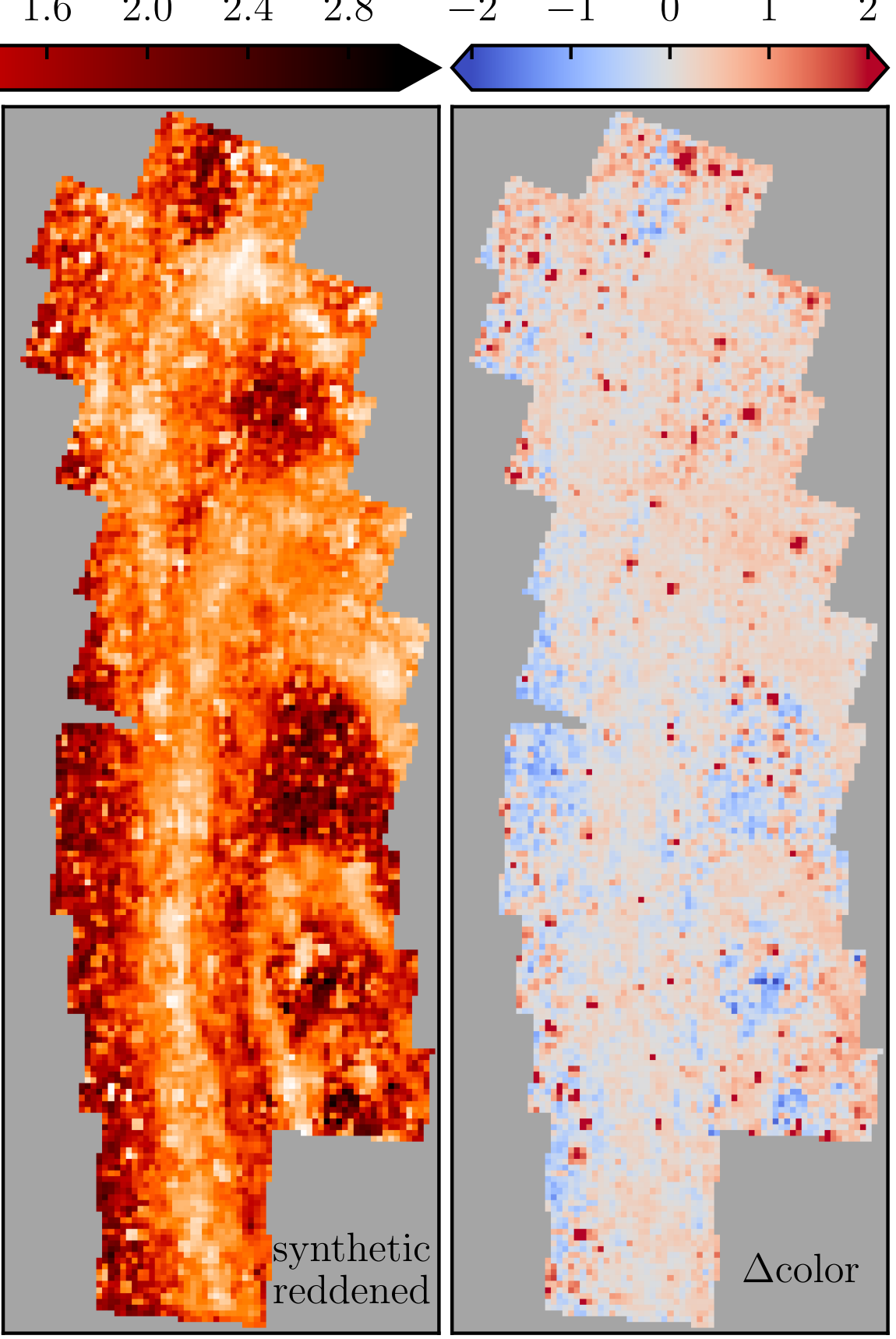

Figure 5. Maps of UV color. The left panel shows the observed color, $\left(m_{\mathrm{FUV}}-m_{\mathrm{NUV}}\right)^{\mathrm{obs}}$, the middle panel shows the modeled, attenuated color, $\left(m_{\mathrm{FUV}}-m_{\mathrm{NUV}}\right)^{\mathrm{syn}}$, and the right panel shows the difference between the two

both the FUV and the NUV fall within $1 \sigma$ of the oneto-one line and $>97 \%$ fall within $3 \sigma$. These percentages do not include the brightest single bin in the FUV and the brightest two bins in the NUV where those numbers are significantly lower. The number of points in each bin as well as the percentage that fall within 1,2 , or $3 \sigma$ are given in Table 3. While we do not match all points perfectly, the degree of agreement is still impressive.

The overall agreement between $f^{\text {syn }}$ and $f^{\text {obs }}$ confirms that our modeling procedure is generally robust and justifies the assumptions we used to model the flux, includ- ing the assumed IMF, stellar models and spectral library, and the extinction model. While the modeling can certainly be made more complex, it is reassuring to know that we can use all of this knowledge to derive SFHs, synthesize SEDs, and successfully recreate detailed maps in the UV, all from photometry in only two optical bands.

\subsection{Issues Affecting the Synthetic Ultraviolet Maps}

The above comparisons strongly support the overall reliability of the SFHs presented in Lewis et al. (2015), particularly the dust parameters derived in the SFH fitting 
Table 3

Fraction of pixels within 1, 2, or 3 Sigma of One-to-One

\begin{tabular}{ccccccccc}
\hline \hline Bin Center & \multicolumn{2}{c}{ N } & \multicolumn{2}{c}{$1 \sigma$} & \multicolumn{2}{c}{$2 \sigma$} & \multicolumn{2}{c}{$3 \sigma$} \\
$\log \left(\mathrm{erg} \mathrm{s}^{-1} \mathrm{~cm}^{-2} \AA^{-1}\right)$ & FUV & NUV & FUV & NUV & FUV & NUV & FUV & NUV \\
\hline-16.65 & 123 & 0 & 0.70 & NA & 0.93 & NA & 0.98 & NA \\
-16.25 & 1518 & 288 & 0.78 & 0.66 & 0.95 & 0.94 & 0.98 & 0.99 \\
-15.87 & 2785 & 3192 & 0.75 & 0.69 & 0.95 & 0.96 & 0.99 & 1.0 \\
-15.47 & 3132 & 4404 & 0.73 & 0.72 & 0.95 & 0.95 & 0.99 & 0.99 \\
-15.08 & 1788 & 1780 & 0.72 & 0.73 & 0.95 & 0.92 & 0.99 & 0.98 \\
-14.68 & 632 & 376 & 0.78 & 0.65 & 0.95 & 0.89 & 0.98 & 0.97 \\
-14.29 & 122 & 66 & 0.70 & 0.48 & 0.92 & 0.82 & 0.99 & 0.92 \\
-13.99 & 12 & 14 & 0.33 & 0.00 & 0.67 & 0.50 & 0.92 & 0.79 \\
\hline
\end{tabular}

Note. - The first column shows the center of the bin of width 0.4 dex. Columns 2 and 3 indicate the number of pixels that fall within that bin. Columns 4 and 5 show the fraction of pixels in that bin that lie within $1 \sigma$ of the one-to-one line (the dashed line in Figure 4). Columns 6 and 7 show the fraction of pixels that lie within $2 \sigma$ of the one-to-one line. Columns 8 and 9 show the fraction of pixels that lie within $3 \sigma$ of the one-to-one line.

process. Therefore, before we proceed to a discussion of the un-reddened maps, we highlight some additional considerations that could affect the modeled flux.

The GALEX data primarily suffers from Poisson uncertainties due to the exposure time. For each of the five DIS images in this study, we assume an average exposure time of $7 \times 10^{3} \mathrm{~s}$ in the FUV channel and $6 \times 10^{4} \mathrm{~s}$ in the NUV channel. The uncertainties are only a few percent at $f^{o b s} \sim 10^{-16} \mathrm{erg} \mathrm{s}^{-1} \mathrm{~cm}^{-2} \AA^{-1}$. The NUV uncertainties are an order of magnitude smaller.

The scatter in Figure 4 is much larger than the above Poisson uncertainties allow. As a result, the scatter seen in Figure 4 is most likely dominated by the modeling process. We now discuss possible sources of uncertainty in our synthetic data.

There are four major factors that could contribute to the scatter observed in the synthetic fluxes: (1) SFH uncertainties, (2) IMF sampling incompleteness, (3) differences between the dust model and the physical properties of the dust, and (4) stellar evolution model uncertainties. Because each of these effects is coupled to the others (e.g., the SFH of each region is dependent on the stellar models used and is also degenerate with the dust in that region), it is difficult to constrain the uncertainties from each source separately to high precision. Instead, we provide a qualitative discussion of each source and its effect on the resulting flux.

\subsubsection{SFH Uncertainties}

We first consider the role of uncertainties in the $\mathrm{SFH}$. At the native time resolution of 0.1 dex, the uncertainties on the SFR in a single time bin can range from $\gtrsim 10$ $100 \%$. The exact number depends on the number of luminous main sequence stars in each region (Lewis et al. 2015), which will affect the certainty of star formation happening in one time bin as opposed to the adjacent time bin. These uncertainties naturally decrease when averaged over larger region sizes or longer timescales.

The uncertainties on the SFR in Lewis et al. (2015) were determined using a hybrid Markov Chain Monte Carlo routine (Duane et al. 1987) to produce a sample of $10,000 \mathrm{SFHs}$. To determine the magnitude of the uncertainties propagated through from the SFHs, we ran 1000 of these SFHs in each region through our SED modeling routine. We take the uncertainty on the flux to be the distribution of fluxes derived from this sample. We note that the uncertainties derived in this manner will be upper limits. Most of the stars that are responsible for the observed UV flux are present in the CMDs, and these stars also account for much of the observed star formation. This is especially true in the FUV, where it is expected that younger populations, which we observe on the CMD, are responsible for most of the observed flux.

In Figure 6, we show the uncertainties in the modeled flux as a function of observed flux for both the reddened and dust-free synthetic flux. The uncertainties are highly asymmetric, especially at the low-flux end because of asymmetries in the SFH uncertainties. When the bestfit SFR in a time bin is zero, the uncertainty on that SFR is only positive. The regions that populate the low-flux end of this figure tend to have little or no star formation in the most recent $100 \mathrm{Myr}$. Over the $100 \mathrm{Myr}$ timescales of interest in this paper, the random uncertainties on the synthetic flux due to the SFH derivation are up to an order of magnitude at the very faint end, though only a factor of a few or less at the bright end. The inset in each subpanel shows the ratio of the total uncertainties shown in Figure 6 to the size of the scatter in Figure 4.

We repeat the same exercise in Figure 7, except we plot the uncertainties as a function of SFR averaged over the most recent 100 Myr. Similar to Figure 6, the uncertainties are asymmetric. As expected, they generally decrease toward higher SFR.

\subsubsection{Dust Models}

We next consider uncertainties in the dust model used to model the SFHs and the flux. As described in Lewis et al. (2015), we modeled dust in the optical CMDderived SFHs with a two-component top-hat model designed to account for constant extinction along the line of sight, and differential extinction internal to the galaxy. The CMD residuals suggest that this model is reasonable for the young stars, but extrapolation of this model to the UV requires assumptions about the attenuation curve and the $2175 \AA$ bump. In the modeling process, we assume that the attenuation curve, $R_{V}$, and the strength of the $2175 \AA$ bump are constant across the disk. However, it is possible that these have spatial variation, and this could lead to scatter in the flux ratios. Robust SED fitters, such as the BEAST (Gordon et al. 2016) may be able to measure such region-to-region variation. Ad- 

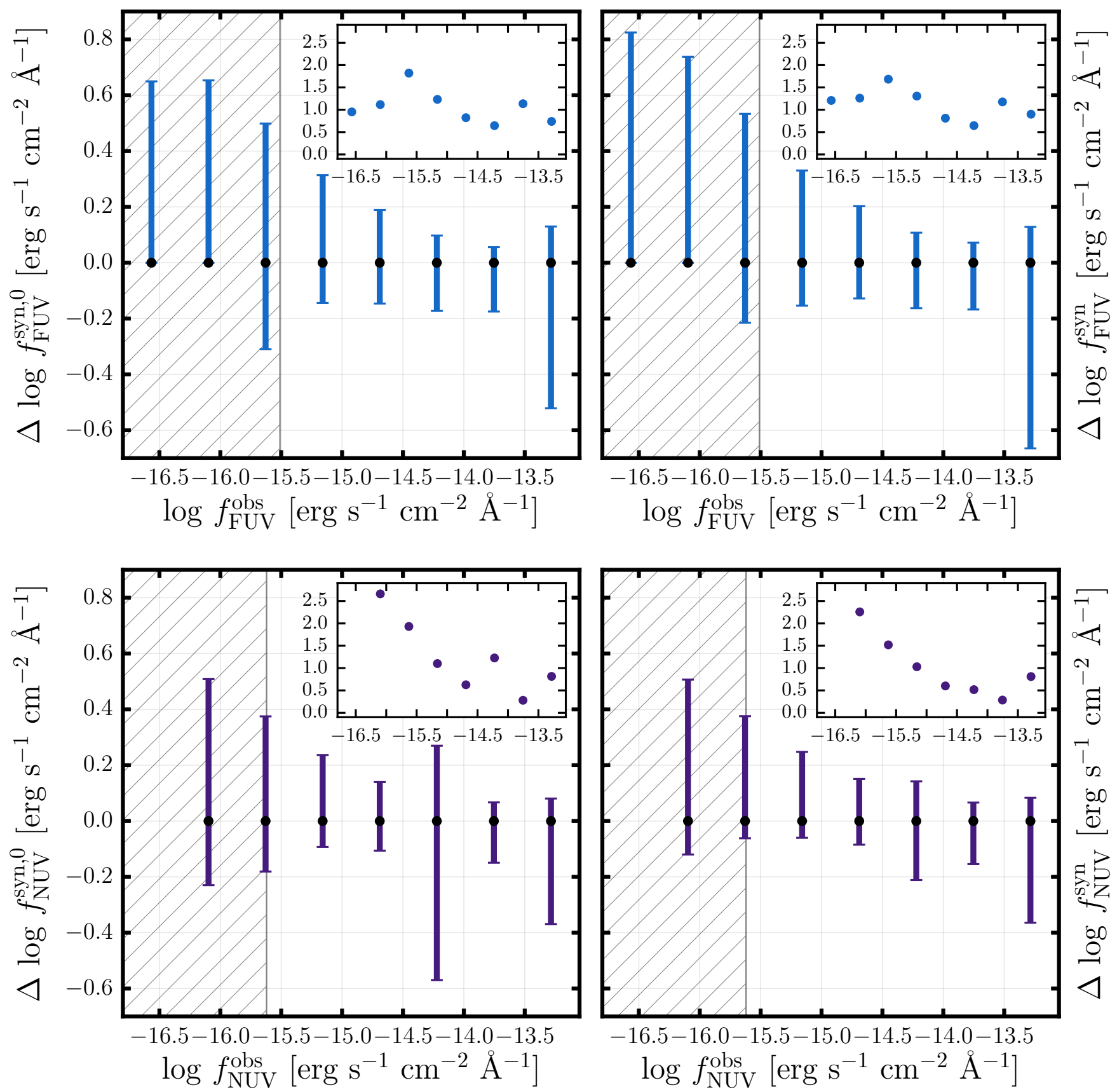

Figure 6. Uncertainties from the SFH derivation on the dust-free and reddened synthetic flux as a function of observed flux. The top panels show the FUV uncertainties and the bottom panels show the NUV uncertainties. Dust-free flux is on the left and reddened flux is on the right. The gray vertical line indicates the total background level (Section 2.1) and therefore the hatched region indicates the flux levels at which GALEX noise takes over. The insets show the ratio of the uncertainties presented in this figure to the scatter shown in Figure 4.

ditionally, scattering of light by dust (Section 4.4) may alter the effective attenuation curve in a given region, producing extra flux that does not originate in that region and giving the attenuation curve a shape that is different from that of a perfectly known extinction curve.

\subsubsection{Incomplete Sampling of the IMF}

A third source of uncertainty is incomplete sampling of the stellar IMF. The UV light in a region is highly dependent on the number of massive stars. In modeling the SFHs, we assumed a fully-sampled IMF. When the
$\mathrm{SFR} \lesssim 10^{-4} \mathrm{M}_{\odot} \mathrm{yr}^{-1}$, however, the IMF may not be fully populated in that there are too few massive stars. This is most likely to occur in regions that are physically small or regions where the SFR is low or non-existent. That said, low flux regions tend to contain mostly older stellar populations. As such, stochastic effects do not make as much of a difference because the UV is coming from stars further down the luminosity function. In high-flux and high-SFR regions, a scale of $30-40 \mathrm{pc}$ (approximately the size of a $4000 \mathrm{M}_{\odot}, 5$ Myr old cluster) appears to be large enough to avoid IMF sampling issues 

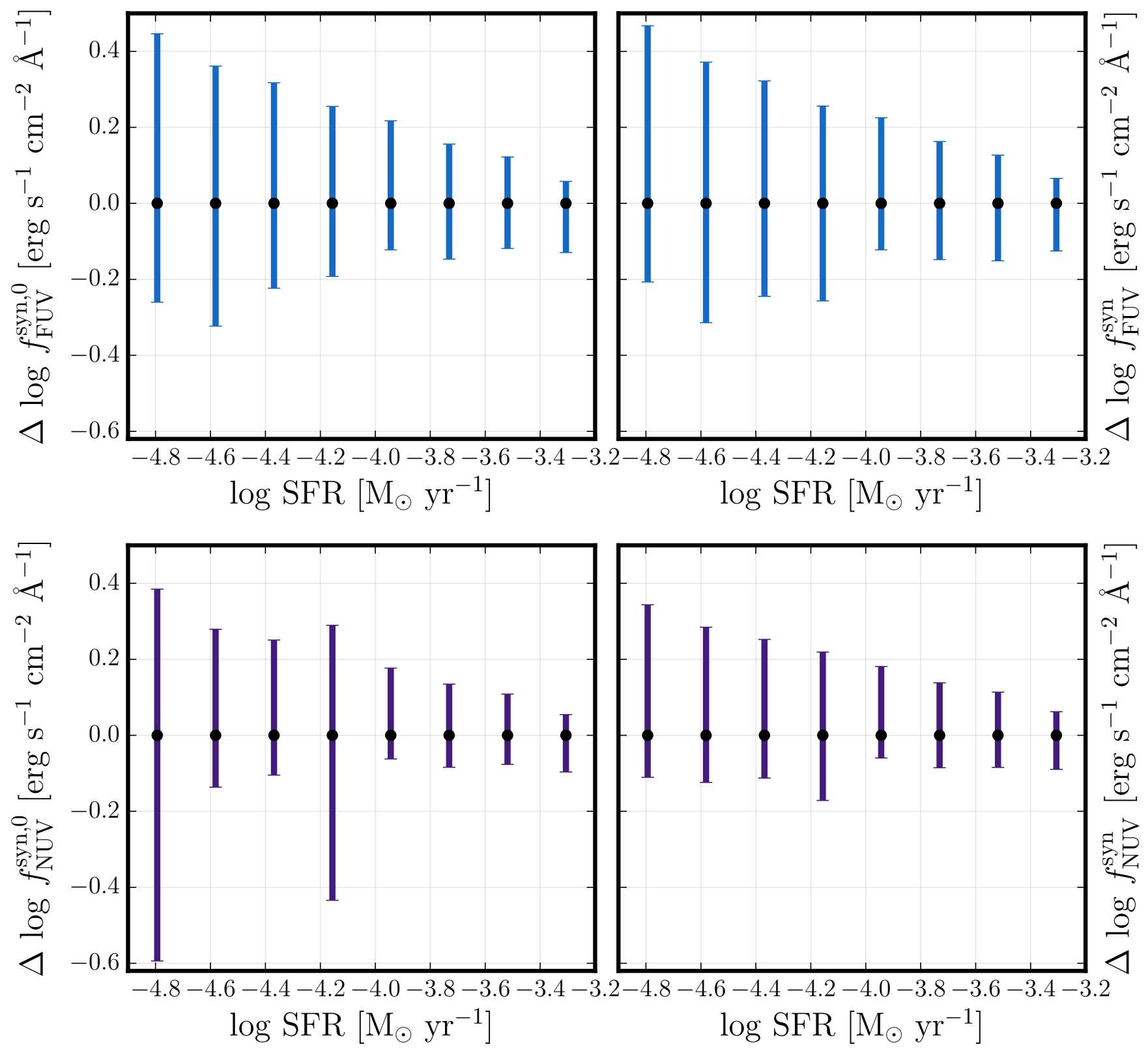

Figure 7. Uncertainties from the SFH derivation on the dust-free and reddened synthetic flux as a function SFR averaged over the last 100 Myr. The top panels show the FUV uncertainties and the bottom panels show the NUV uncertainties. Dust-free flux is on the left and reddened flux is on the right. We have only included SFRs above $10^{-5} \mathrm{M}_{\odot} \mathrm{yr}^{-1}$. Uncertainties at lower SFRs (not on this figure) are $0.8-1.0$ dex in the FUV and $0.4-0.5$ dex in the NUV.

(Boquien et al. 2015). Additionally, several studies have shown that stochastic sampling of the IMF - even at very low SFR - has less impact on the FUV and NUV fluxes because they result from integration over a wide mass range and are less dependent on the most massive stars than an ionizing luminosity such as $\mathrm{H} \alpha$ (e.g., Lee et al. 2009, 2011; da Silva et al. 2012; Johnson et al. 2013; da Silva et al. 2014).

In general, the $100 \mathrm{pc} \times 400 \mathrm{pc}$ regions in this study are more than large enough to avoid effects of stochastic sampling in high SFR regions. In lower SFR regions, stochastic sampling could potentially affect our results. However, incomplete sampling of the IMF does not overwhelmingly contribute to the observed scatter in the flux ratios. The FUV and NUV fluxes are integrated over a stellar mass that reaches down to $\sim 3-5 \mathrm{M}_{\odot}$ on the main sequence. Additionally, stochastic effects of the $\mathrm{IMF}$ are incorporated into the uncertainties on the SFHs as described in Lewis et al. (2015). Consequently, it is unlikely that incomplete sampling of the IMF contributes significantly to the scatter in the flux ratios beyond the uncertainties already accounted for in the SFH measurements.

\subsubsection{Stellar Models}

The last source of uncertainty is from deficiencies in the stellar models used to derive the SFHs and to model the flux. Just as the number of massive stars affects the observed UV light, discrepancies between the synthetic and true UV properties of these massive, metal rich stars 
could introduce scatter into the flux ratios. This uncertainty is the most challenging to quantify. The fidelity of the stellar models will be the same in all locations, but the impact of the models will vary from region to region due to variation with stellar type and mass and therefore with SFH and IMF sampling. Perhaps most importantly, stellar models in the UV are poorly calibrated due to the paucity of massive, metal-rich stars in the local universe.

\subsubsection{Summary of Uncertainties}

Based on the above effects, the scatter in the flux ratios shown in Figure 4 is dominated by the uncertainties in the SFH, which include scatter due to incomplete sampling of the IMF.

\subsection{Emission Timescales}

Fluxes in the FUV and NUV are often attributed to timescales of $<100 \mathrm{Myr}$ and $<300 \mathrm{Myr}$, respectively, because most of the UV emission comes from the hottest, most massive stars that dominate younger stellar populations (Kennicutt \& Evans 2012). It has also been shown that older populations emit in the UV as well (e.g., O'Connell 1999), with often as much as $20-30 \%$ of the total emission in the FUV or NUV coming from these stars (e.g. Johnson et al. 2013). The contribution from older populations is generally negligible in regions of high recent star formation. However, in regions of low SFR, this contribution can be non-negligible, particularly in the NUV. Unfortunately, the CMD modeling in Lewis et al. (2015) excluded regions of the CMD in the fit that are most sensitive to old stellar populations, and thus our ability to model the UV contribution from older star formation is limited. Including this contribution would be most likely to increase the low SFR intensity region fluxes while leaving the high SFR intensity regions unaffected.

\subsection{Scattered Light}

A portion of a galaxy's UV light can be diffuse and not associated with an obvious star-forming region. This diffuse UV emission is found between visible spiral arms or rings. In some cases, this fraction can be quite significant (up to $65 \%$ in M33; Thilker et al. 2005). There are a number of suggestions for the origination of this diffuse light, including the dispersal of B-type stars after the dissolution of their natal clusters, a low level of diffuse star formation, or the scattering by dust of UV photons produced in bright star-forming regions (Marcum et al. 2001; Crocker et al. 2015).

This scattered light may redistribute the flux in the observed UV maps in a manner that is not captured in our synthetic maps. We can, however, use our modeled data to examine the extent of scattered light in M31. The right panel in Figure 2 shows the log ratio of the observed to the modeled reddened FUV flux. Blue pixels have more synthetic flux than observed with GALEX and red pixels have more observed flux than synthetic flux. We have predicted slightly more synthetic flux than is observed (blue pixels) in just over half of the regions. However, the regions in which we have under-predicted the flux have a larger offset than the regions in which we have over-predicted the flux. This excess GALEX flux occurs primarily along the eastern side of the $10 \mathrm{kpc}$ ring as well as near two large OB associations in Bricks 15 \& 21. This discrepancy is particularly interesting because it is found near regions of very high, recent star formation. The many star-forming regions along the northeastern section of the ring are responsible for the bump in the star formation rate seen $\sim 50 \mathrm{Myr}$ ago (Lewis et al. 2015). The excess observed flux seen near these highly star-forming regions could be an indication that some of this UV light has been scattered by dust into regions where no star formation is occurring. Further analysis of scattered light is beyond the scope of this paper.

\subsection{Comparison with PHAT F275W data}

The PHAT survey consists of data in six filters, two each in the NUV, the optical and the near-infrared. In the SFH derivation (Lewis et al. 2015), we used only the optical data as they are the deepest and provide the greatest leverage on the recent SFH. In theory, the ultraviolet filters (F275W and F336W) would also provide important constraints on the recent SFH. In practice, only the brightest main sequence stars have measurements in the UV filters, which greatly reduces the age range over which the SFH can be derived. Therefore, the UV filters were not used in the SFH derivation.

We can, however, compare the PHAT UV data, in particular the F275W data, with the synthetic, reddened NUV maps derived from the optical data. In Figure 8, we show several maps of B15 including the synthetic, reddened NUV flux (top, left), the internal reddening derived from the $\mathrm{SFH}$ fitting process, $d A_{\mathrm{V}, \mathrm{SFH}}$ (top, right), and the F $275 \mathrm{~W}$ flux in individually detected stars, both gridded to the same scale as the UV maps (bottom, left) and at the individual star resolution (bottom, right). The synthetic NUV and F275W flux images are colored according to the log of the flux in each band.

We chose B15 because it sits on the $10 \mathrm{kpc}$ ring and therefore contains both active star formation and dust. The most obvious region of star formation is the bright region in the lower right of the brick (OB 54; van den Bergh 1964). In Figure 8, both the synthetic NUV and the gridded F275W flux in the left panels show a region of high flux (yellow in color) in that region. In the bottom right panel, the density of stars detected in $\mathrm{F} 275 \mathrm{~W}$ is much greater in that region and along the ring feature than in other parts of the brick.

The F275W data comes from the gst catalog, which contains only stars with reliable measurements (high signal-to-noise and low crowding) in each band. We chose to use the individually resolved stars rather than the full brick images of B15 for a number of reasons. There are only two exposures per UVIS band, making it difficult to reliably clean the images of cosmic rays (CRs). By using the gst catalog of resolved stars with its more stringent cuts, we avoid artifacts due to CRs. There are also problems due to charge transfer efficiency (CTE) because CTE corrections were not available for WFC3. Finally, as noted above, the images are shallow.

We have avoided some of the above problems by using the resolved stars; however, there are limitations to this choice as well. In particular, we cannot include stars below the flux limit that were not detected. Additionally, while using the gst catalog eliminates artifacts, it may also remove real detections. Finally, in the UVIS images, recovery of stars is biased such that artificial stars at the 
faint end are recovered at fainter magnitudes than those at which they are placed. We refer the reader to Williams et al. (2014) for details on the photometric catalog. As a result, the bottom two panels in Figure 8 are not complete, but they are adequate for purposes of qualitative comparison.

A detailed region-by-region comparison of the synthetic, reddened NUV image and the gridded F275W image cannot be made due to differences in wavelength and the lack of completeness in the F275W stellar catalog, as discussed above. However, the two images are qualitatively very similar, and these similarities (and differences) may be important for understanding the distribution and morphology of the young, massive stars responsible for the bulk of the NUV emission. Further analysis would require additional modeling, which is beyond the scope of this paper.

\section{RESULTS}

There are many different uses for the maps we have presented in this paper, including testing standard procedures for understanding the fraction of obscured star formation as well as the the applicability of common SFR prescriptions. In this section, we compare standard corrections for dust with results from the synthetic flux to better understand SFR limitations.

\subsection{Obscured Flux and Star Formation}

In Section 4, we presented synthetic GALEX maps and verified that they were in superb agreement with observations, especially in the FUV where the impact of the "2175 A bump" is negligible. We now consider the un-reddened synthetic images $\left(f^{\text {syn, } 0}\right)$ and compare them to standard estimates of the fraction of obscured star formation. We note that because FUV flux maps directly to SFR in a galaxy, we are using the fraction of FUV flux that is obscured by dust as a proxy for the fraction of obscured star formation. We will convert to SFR and discuss the effects of the obscured flux in Section 5.2.

As already discussed, the FUV is an ideal tracer of star formation because it is tied directly to emission from the youngest and brightest $\mathrm{O}$ and B-stars. It is also highly affected by dust. Use of the FUV as a monochromatic SFR tracer underestimates the true SFR of a galaxy. It has long been noted, though, that a correction could be made by including the flux from the longer wavelengths where dust emits. Early studies suggested using the total infrared emission

Over the past several years, it has become standard to estimate the amount of obscured star formation by "correcting" the observed emission from young stars with the observed $24 \mu \mathrm{m}$ flux from Spitzer. The idea is essentially one of energy balance. In a dust-free region, the total SFR can be inferred from the observed emission from young stars. In dusty regions, some of that emission is absorbed by the dust. The total flux of light that reaches the telescope is reduced and the derived SFR will be under-estimated. The dust obscures the star formation. However, the emission absorbed by the dust is not lost but rather re-emitted at different, longer wavelengths. Therefore, if we include that longer wavelength emission in our roundup of the total flux, the true SFR can be accurately derived.
Calzetti et al. (2007) first used $24 \mu \mathrm{m}$ emission as a correction to $\mathrm{H} \alpha$ flux. They observed a correlation between the luminosity surface densities of $\mathrm{Pa} \alpha$ emission and $24 \mu \mathrm{m}$ emission. Pa $\alpha$ emission traces the ionizing photons from the photospheres of hot, young stars and is significantly less affected by dust than $\mathrm{H} \alpha$, making it an ideal tracer of star formation in a galaxy (e.g., Kennicutt 1998). $24 \mu \mathrm{m}$ emission traces the thermal dust emission of a galaxy originating from small dust grains (e.g., Draine \& Li 2007). Calzetti et al. (2007) combined $\mathrm{H} \alpha$ emission with $24 \mu \mathrm{m}$ emission and compared the result with the $\mathrm{Pa} \alpha$ emission to derive a calibration for a $\mathrm{H} \alpha+$ $24 \mu \mathrm{m}$ SFR. Following this result, Leroy et al. (2008) derived a similar relation using FUV emission rather than $\mathrm{H} \alpha$ emission. Rather than using $\mathrm{Pa} \alpha$ emission for the total SFR, they estimated the scaling factor on $24 \mu \mathrm{m}$ using various estimates of the total SFR. Similarly, Hao et al. (2011) used integrated measurements of the Moustakas \& Kennicutt (2006) sample of nearby, star-forming galaxies to compare variations of UV + monochromatic infrared luminosities with IRX (total infrared to FUV luminosity ratio)-corrected FUV luminosities and derive composite SFR tracers, including a FUV $+25 \mu \mathrm{m}$ tracer.

We note that, in this paper, we are interested only in the $24 \mu \mathrm{m}$ correction to the SFR. The idea of correcting the FUV luminosity for dust using FIR emission goes back to at least the late 1990s (e.g., Meurer et al. 1999; Gordon et al. 2000; Bell \& Kennicutt 2001).

Dust obscuration is an extremely variable quantity. Non-active Sb-Scd galaxies typically lose about half of their bolometric luminosity to dust absorption. In earlytype galaxies, this quantity drops to less than $15 \%$ Calzetti (2001). In M33, Boquien et al. (2015) found $75 \%$ of star formation in $\mathrm{H} \alpha$, with only $25 \%$ in the infrared.

In Figure 9, we combine our synthetic FUV flux maps to examine the fraction of obscured star formation within the PHAT footprint and compare them with corresponding observed data from GALEX and Spitzer. In the left column, we show the synthetic maps derived in this paper. In the top row, we plot the synthetic, reddened flux, $f_{\text {FUV }}^{\text {syn }}$. The middle row shows the dust-corrected flux, i.e., the synthetic, dust-free flux $f_{\mathrm{FUV}}^{\mathrm{syn}, 0}$. These two maps are the same as those presented in Figure 2 but with a different scaling to emphasize the dust-free map. In the bottom row, we plot the fraction of obscured FUV flux using the images in the first two rows: $1-f_{\mathrm{FUV}}^{\mathrm{syn}} / f_{\mathrm{FUV}}^{\mathrm{syn}, 0}$.

The increase in total flux in the ring features immediately jumps out. Most of the star formation takes place in the $10 \mathrm{kpc}$ ring (outlined in blue Lewis et al. 2015), which is also the dustiest part of the galaxy. We would therefore expect to see the most change in the flux in the ring features. The width of the ring also increases, indicating that the regions on the edges of the rings are also dusty. Overall, we find that in the synthetic dustfree data there is a factor of 8 more total flux than in the synthetic reddened data. If we look at the ring features individually, the factor difference between the total flux is $7.3,8.2$, and 7.8 , for the inner, $10 \mathrm{kpc}$, and outer ring features respectively. The median factor increase for all pixels in each region is 5.6, 5.1, and 4.6, respectively.

Because M31 is a very well-studied galaxy, a lot of ancillary data exists. In addition to the $G A L E X$ images, we also have $24 \mu \mathrm{m}$ images from Spitzer. We can therefore 


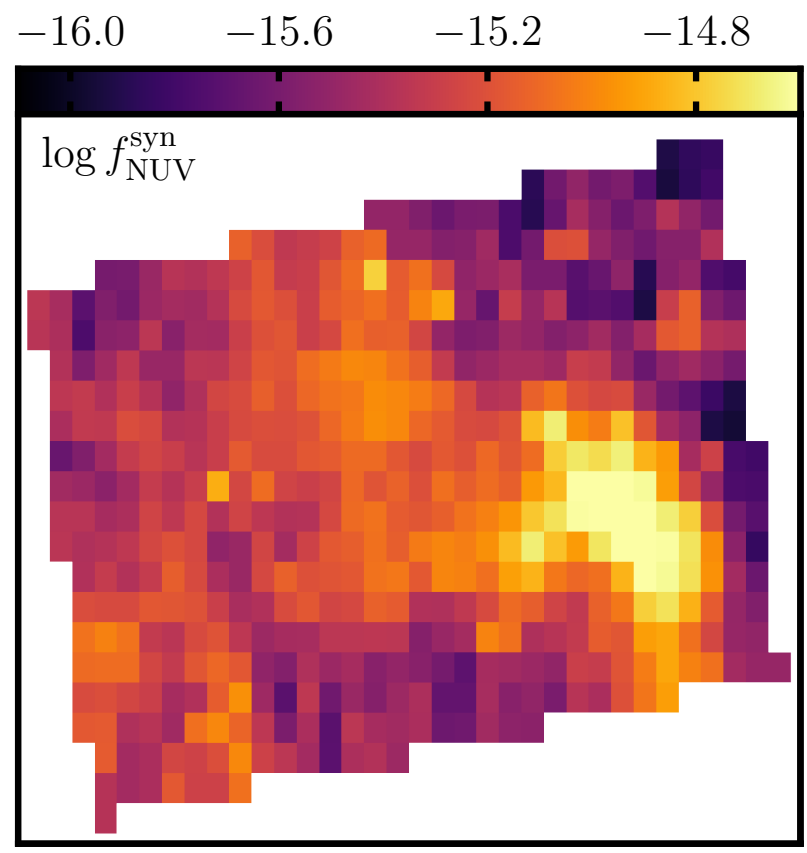

\section{$\begin{array}{llllllll}0.1 & 0.4 & 0.7 & 1.0 & 1.3 & 1.6 & 1.9 & 2.2\end{array}$}
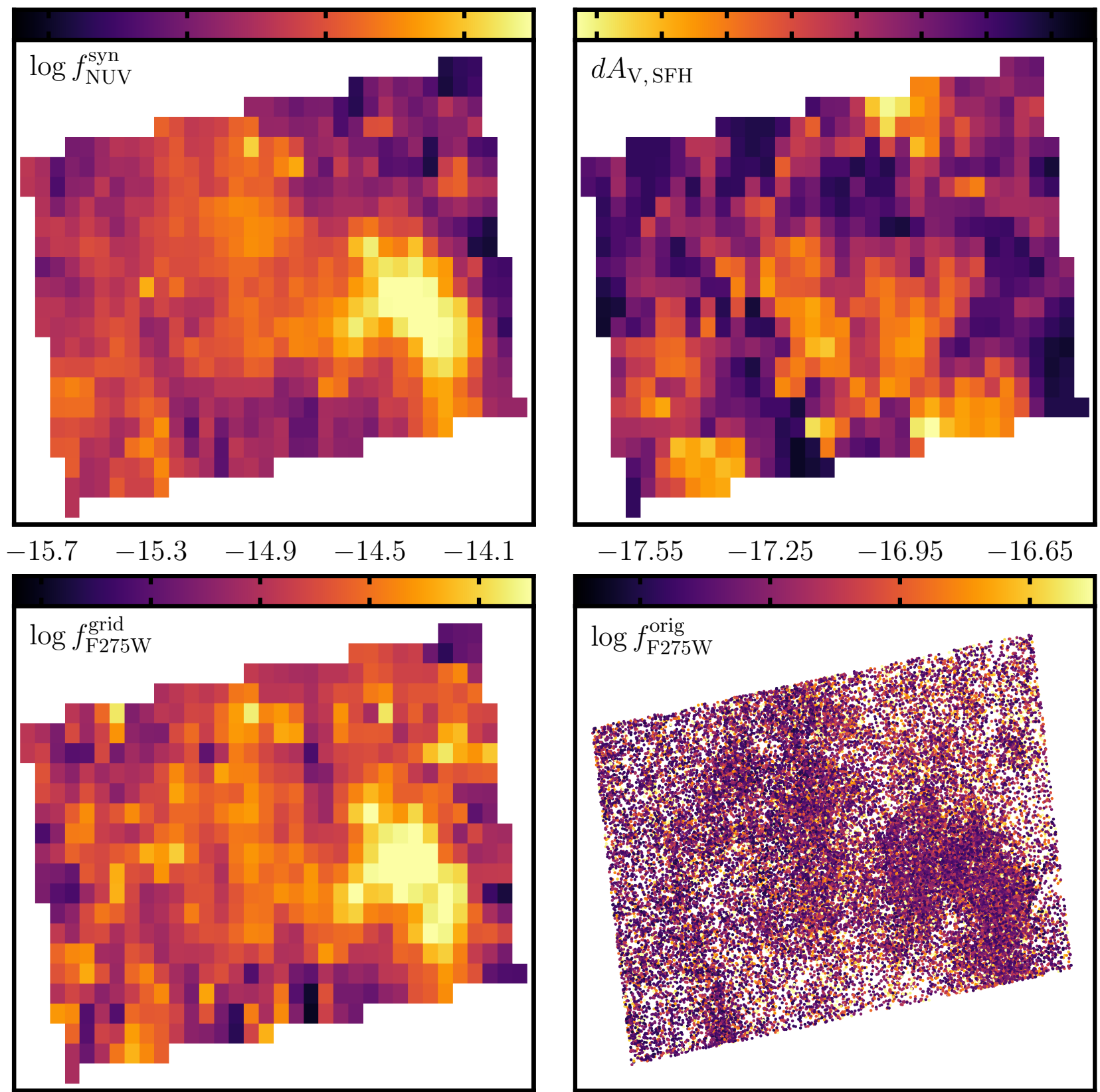

Figure 8. Comparison of synthesized NUV data with PHAT F275W gst data in B15. Synthetic, reddened NUV image (top, left), optimized internal reddening parameter determined from the SFH derivation, $d A_{\mathrm{V}} \mathrm{SFH}$ (top, right), PHAT F275W flux from individual stars binned to 100 pc (bottom, left), PHAT F275W flux, individual stars (bottom, right).

compare our obscured fraction analysis with estimates from other observables. We have already compared the synthetic reddened data to the GALEX FUV image. We can also compare the synthetic dust-free data to a derived GALEX FUV + Spitzer $24 \mu \mathrm{m}$ image. We use the prescriptions from Hao et al. (2011) to correct the FUV image for dust:

$$
L(\mathrm{FUV})_{\mathrm{corr}}=L(\mathrm{FUV})_{\mathrm{obs}}+3.89 \times L(25 \mu \mathrm{m}) .
$$

All luminosities have units of erg $\mathrm{s}^{-1}$.

In the right column of Figure 9, we present corresponding maps of traditional observables of $\mathrm{FUV}+24$ $\mu \mathrm{m}$ (e.g., Hao et al. 2011; Kennicutt \& Evans 2012).
In the top row, we plot the observed GALEX flux, $f_{\mathrm{FUV}}^{\text {obs }}$. The middle row shows the dust-corrected flux, i.e., the GALEX FUV flux corrected with $24 \mu \mathrm{m}$ flux, $f_{\mathrm{FUV}}+24 \mu \mathrm{m}$. In the bottom row, we plot the fraction of obscured flux using the images in the first two rows. In panel (f) we use the usual observables: 1 - $f_{\mathrm{FUV}}^{\text {obs }} /$ $f_{\mathrm{FUV}+24 \mu \mathrm{m}}$. The $24 \mu \mathrm{m}$ data comes from Gordon et al. (2006).

While the synthetic reddened and the GALEX maps look very good (total flux is conserved within $8 \%$ ), the dust-free maps are quite different. There is a factor of 2.5 more flux in the synthetic map than in the GALEX $+24 \mu \mathrm{m}$ map. Overall, we find that $88 \%$ of the flux is 


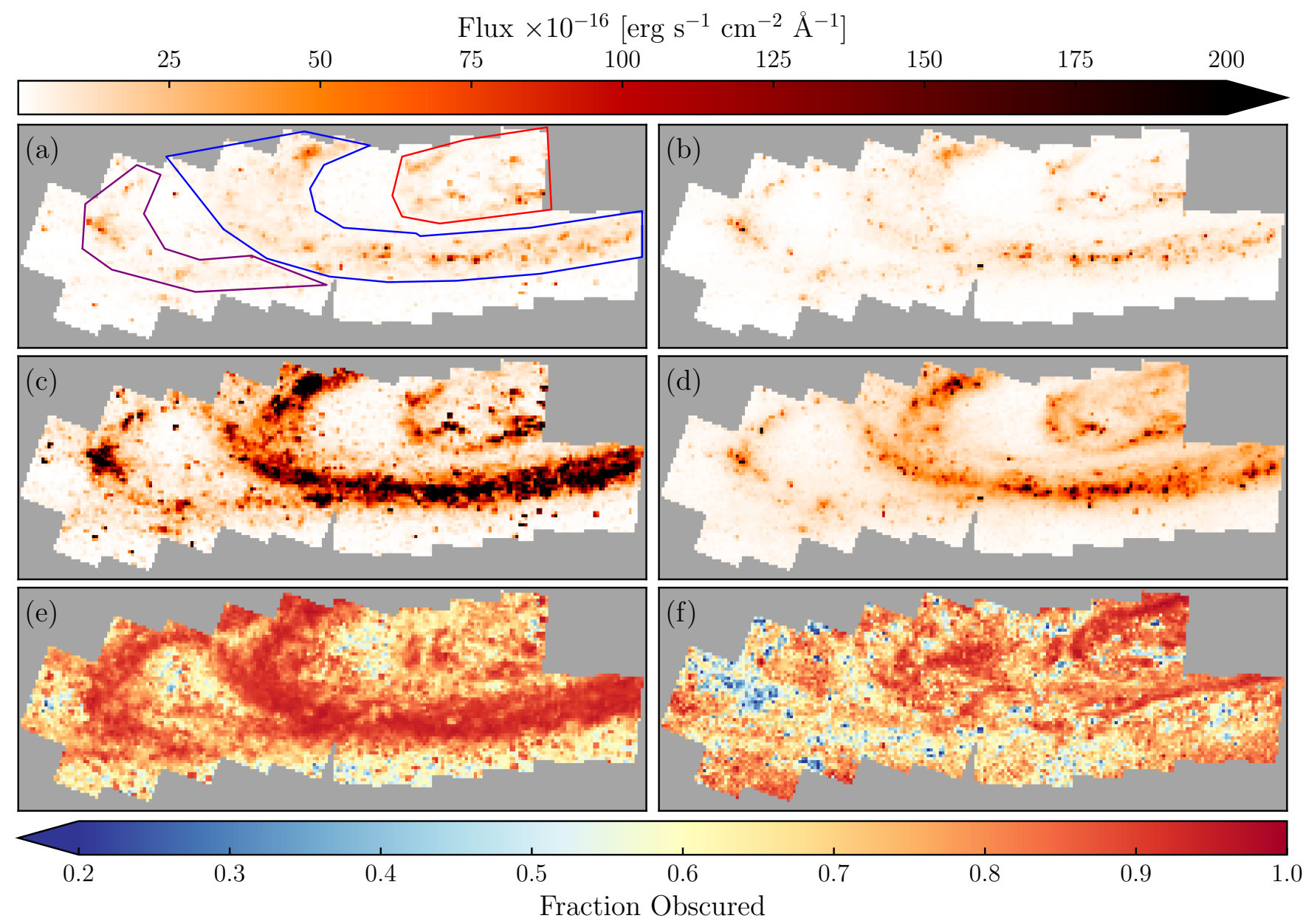

Figure 9. Obscured Flux and Star Formation. (a) Synthetic, reddened FUV flux: $f_{\mathrm{FUV}}^{\text {syn }}$, (b) observed GALEX FUV flux: $f_{\mathrm{FUV}}^{\text {obs }}$, (c) synthetic, un-reddened FUV flux: $f_{\mathrm{FUV}}^{\text {syn, }},(\mathrm{d})$ observed dust-correct flux: $f_{\mathrm{FUV}}+24 \mu \mathrm{m}$, (e) fraction of obscured flux from the synthetic maps: $1-f_{\mathrm{FUV}}^{\mathrm{syn}} / f_{\mathrm{FUV}}^{\mathrm{syn}, 0}$, (f) fraction of obscured flux from traditional observables: $1-f_{\mathrm{FUV}}^{\mathrm{ob}} / f_{\mathrm{FUV}}+24 \mu \mathrm{m}$. Panels (a) - (d) are on the same flux scale and panels (e) and (f) are on the same scale for ease of comparison. In panel (a), we have outlined the ring features in blue (10 kpc ring), red (inner), and purple (outer). While panels (a) and (b) show remarkable agreement, the dust-corrected flux in panels (c) and (d) looks very different. In particular, the synthetic, dust-free maps indicate much more star formation in the ring and outer arm. The difference between the dust-free maps causes the fraction of obscured flux (maps (e) and (f)) to be structurally distinct. Panel (e), which uses the synthetic data, indicates that the $10-\mathrm{kpc}$ ring and the $15 \mathrm{kpc}$ ring feature are heavily dust obscured, while panel (f) suggests that only parts of the 10-kpc ring are obscured but that much more of the inner ring feature is enshrouded.

obscured in our synthetic maps, while $72 \%$ is obscured in the observed maps. The $24 \mu \mathrm{m}$ correction therefore under-estimates the total FUV flux. This has a direct effect on the quantities derived from that flux, including the SFR.

We now look at the individual features of the maps. The first thing to notice is the difference in the $10 \mathrm{kpc}$ ring between the two maps. If we sum up the total flux in that region, the synthetic map has a factor of 2.5 more flux than the observed map, although the median factor of the individual pixels is only 1.4. While both maps show structure within the ring, the primary difference is that the synthetic map implies that there is more flux at almost all locations. In the inner region of the galaxy, the overall structure is very similar in both maps, but the synthetic map contains a factor of 2.6 more flux, with a median of 1.9. Finally, the outer ring feature follows the same pattern. The overall structure is very similar, but the total flux is 2.4 times higher in the synthetic map with a median factor of 1.2. These differences show that in star-forming, dusty regions, the synthetic dust-free map reveals more flux than the $24 \mu \mathrm{m}$-corrected FUV map.

While the interarm regions lack the coherent structure of the ring features, the flux comparison in the synthetic and observed maps shows a similar trend. The total flux in the interarm regions of the synthetic map is a factor of 2.5 times higher than that in the observed map with a median value of 1.3 .

The differences discussed above lead directly to the distinction in the maps of the fraction of obscured flux in the bottom row of Figure 9. As already discussed, the synthetic maps require a high degree of obscuration. Most of this dust occurs in the ring features, which are strong features in the obscured fraction map. However, all pixels have dust and are therefore obscured. The minimum and maximum obscuration in the synthetic maps is $19 \%$ and $97 \%$, respectively.

There is much less structure in the $24 \mu \mathrm{m}$-based obscured fraction map. In this map, the most obscured regions are towards the center of the galaxy. The $10 \mathrm{kpc}$ ring and outer ring feature are less apparent while the in- 
ner ring feature is stronger. Additionally, the inner edge of each ring feature is more obscured than the feature itself; i.e., it is still possible to pick out the $10 \mathrm{kpc}$ ring in the $24 \mu \mathrm{m}$ obscured fraction map, though only at one edge. Finally, we note that the bright star-forming region in B21 (the bright feature at left edge of panel c) has very little obscuration in the observed data suggesting that the stars in this region are old enough to have dispersed their natal cloud but have not yet reached more evolved, dusty stages. Our results show that the $24 \mu \mathrm{m}$ correction for dust does not work well in regions of low star formation intensity which are likely dominated by older stellar populations.

\subsection{SFR Measurements}

The discrepancy between the synthetic dust-free flux map and the FUV $+24 \mu \mathrm{m}$ flux map indicates that the overall SFR will also be affected. The usual method of converting a FUV flux into a SFR is to apply an extinction correction to the observed flux, convert the flux to luminosity, and then calculate a SFR according to a standard calibration. A common calibration is that from Kennicutt (1998) with updates by Hao et al. (2011) and Murphy et al. (2011):

$$
\mathrm{SFR}=10^{-43.35} \times L_{\mathrm{FUV}} .
$$

where $L_{\mathrm{FUV}}$ is the dust-corrected FUV luminosity with units of erg $\mathrm{s}^{-1}$ and the resulting SFR has units of $\mathrm{M}_{\odot} \mathrm{yr}^{-1}$.

There are a variety of methods for correcting the data for dust. As mentioned in Section 5.1, a popular method is to multiply the $24 \mu \mathrm{m}$ flux by a constant, $w$, especially in relatively nearby galaxies where high quality Spitzer $24 \mu \mathrm{m}$ data exists. Another method is to use UV color $\left(m_{\mathrm{FUV}}-m_{\mathrm{NUV}}\right)$ to correct the FUV magnitude which is then converted to flux for further conversion to SFR. This method is often used at high redshift.

We use the calibrations from Hao et al. (2011). The correction using $24 \mu \mathrm{m}$ data is shown in Equation 4. The $\mathrm{UV}$ color correction is:

$$
\begin{aligned}
& A_{\mathrm{FUV}}= \\
& \quad(3.83 \pm 0.48)\left[(\mathrm{FUV}-\mathrm{NUV})_{\mathrm{obs}}-(0.022 \pm 0.024)\right] .
\end{aligned}
$$

In Figure 10, we examine the relationship between the SFR averaged over the last $100 \mathrm{Myr}$ as calculated from the CMD-derived SFH (Lewis et al. 2015) and that derived from FUV flux corrected for extinction with the 24 $\mu \mathrm{m}$ or UV color calibrations described above. In each panel, we plot the CMD-derived SFR ( $\left.\mathrm{SFR}_{\mathrm{CMD}}\right)$ on the $\mathrm{x}$-axis. The top panels show the flux-to-flux relationship. In the bottom panels, we plot the ratio of the flux-based SFR to the cmd-based SFR ( $\left.\mathrm{SFR}_{\text {flux }} / \mathrm{SFR}_{\mathrm{CMD}}\right)$ on the y-axis. In each panel, the black dashed line represents one-to-one agreement. In the top panels, the black star marks the flux-weighted mean along each axis. In the bottom panels, we have plotted the running median and standard deviation in blue.

In the left panels, we show the flux-based SFR $\left(\mathrm{SFR}_{\text {flux }}\right)$ derived after using the $24 \mu \mathrm{m}$ prescription to correct for extinction. In the right panels, we show the flux-based SFR calculated from FUV flux corrected for dust with the UV color calibration.

We first look at the left panels. On the top we plot the SFR derived from the $24 \mu$ m-corrected FUV flux against the CMD-derived SFR averaged over the most recent $100 \mathrm{Myr}$. The overall morphology of the data is ok using the $24 \mu \mathrm{m}$ correction. There is a clear trend in the data that $\mathrm{SFR}_{\mathrm{flux}, 24 \mu \mathrm{m}}$ increases as $\mathrm{SFR}_{\mathrm{CMD}}$ increases, as is expected. The flattening in $\mathrm{SFR}_{\text {flux }, 24 \mu \mathrm{m}}$ occurs at $\mathrm{SFR}_{\mathrm{CMD}}<\sim 10^{-6}$ which corresponds approximately to the limit at which the flux calibrations are no longer reliable, especially on these spatial scales (e.g., Murphy et al. 2011; Leroy et al. 2012; Kennicutt \& Evans 2012). This results in an over-estimate of the SFR at low $\mathrm{SFR}_{\mathrm{CMD}}$ and an under-estimate at high $\mathrm{SFR}_{\mathrm{CMD}}$. This trend is seen more clearly in the bottom left panel, where we plot the $\log$ of the ratio between $\mathrm{SFR}_{\text {flux }, 24 \mu \mathrm{m}}$ and $\mathrm{SFR}_{\mathrm{CMD}}$. The over-estimate of $\mathrm{SFR}_{\mathrm{flux}, 24 \mu \mathrm{m}}$ increases as $\mathrm{SFR}_{\mathrm{CMD}}$ decreases. At higher $\mathrm{SFR}_{\mathrm{CMD}}$, the ratio starts to flatten though offset from the one-to-one line.

While the overall morphology of the relationship between $\mathrm{SFR}_{\text {flux }}$ and SFR $\mathrm{CMD}$ is good, the flux-weighted mean, marked by the black star in the top left panel, is offset. We find that the mean flux-based SFR is 0.39 dex lower than the mean CMD-based SFR. The 24 $\mu$ m-corrected FUV flux will therefore under-estimate the SFR by a factor of $\sim 2.5$.

The right panels in Figure 10 show the comparison between $\mathrm{SFR}_{\mathrm{CMD}}$ and $\mathrm{SFR}_{\mathrm{flux}, \mathrm{FUV} \text {-NUV. The results are }}$ very different from the $24 \mu \mathrm{m}$ correction. When using UV color, the morphology of the relationship is completely wrong. On a region-to-region basis, there is zero correlation between $\mathrm{SFR}_{\mathrm{CMD}}$ and $\mathrm{SFR}_{\text {flux,FUV-Nuv. Any }}$ SFR $_{C M D}$ can map to a two order of magnitude range in SFR $_{\text {flux,FUV-NUV. The only trend that exists is that }}$ the UV color correction results in an over-estimate of SFR $_{\text {flux,FUV-NUV }}$ at low SFR $_{\text {CMD }}$ and an under-estimate at high $\mathrm{SFR}_{\mathrm{CMD}}$. Despite the absence of region-to-region correlation, the flux-weighted mean SFR falls directly on the one-to-one line.

We note that we could have used the synthetic, dustfree FUV flux-derived SFR on the x-axis instead of the 100 Myr averaged SFR from the CMD-derived SFHs. The results would be the same with an offset of the mean flux-weighted SFRs from the one-to-one line when using the $24 \mu \mathrm{m}$ correction and a lack of correlation when using the UV color correction.

\section{DISCUSSION}

We have shown that the conversion of FUV flux into a SFR after correcting for dust with two different prescriptions results in vastly different relations when compared with the SFR determined from the CMD-derived SFH (or alternatively from using Equation 5 on the dust-free synthetic FUV flux, $f_{\mathrm{FUV}}^{\mathrm{syn}, 0}$ ). These differences are perhaps not surprising, but they do beg the question of what drives the discrepancies. At the most basic level, the discrepancy arises either from the modeled data (synthetic flux or CMD-based SFR) or from the SFR calibrations in the literature.

As discussed in Lewis et al. (2015) and Section 4.2, there are a variety of factors that affect the CMD-derived SFHs and the synthetic flux. The primary uncertainties come from the chosen dust parameters and uncertainties in the SFHs, which were only of order $10 \%$ over a 100 Myr timescale. We also showed in Section 4 and Figure 4 that the synthetic, reddened flux is in very good agreement with the observed GALEX FUV flux. This 


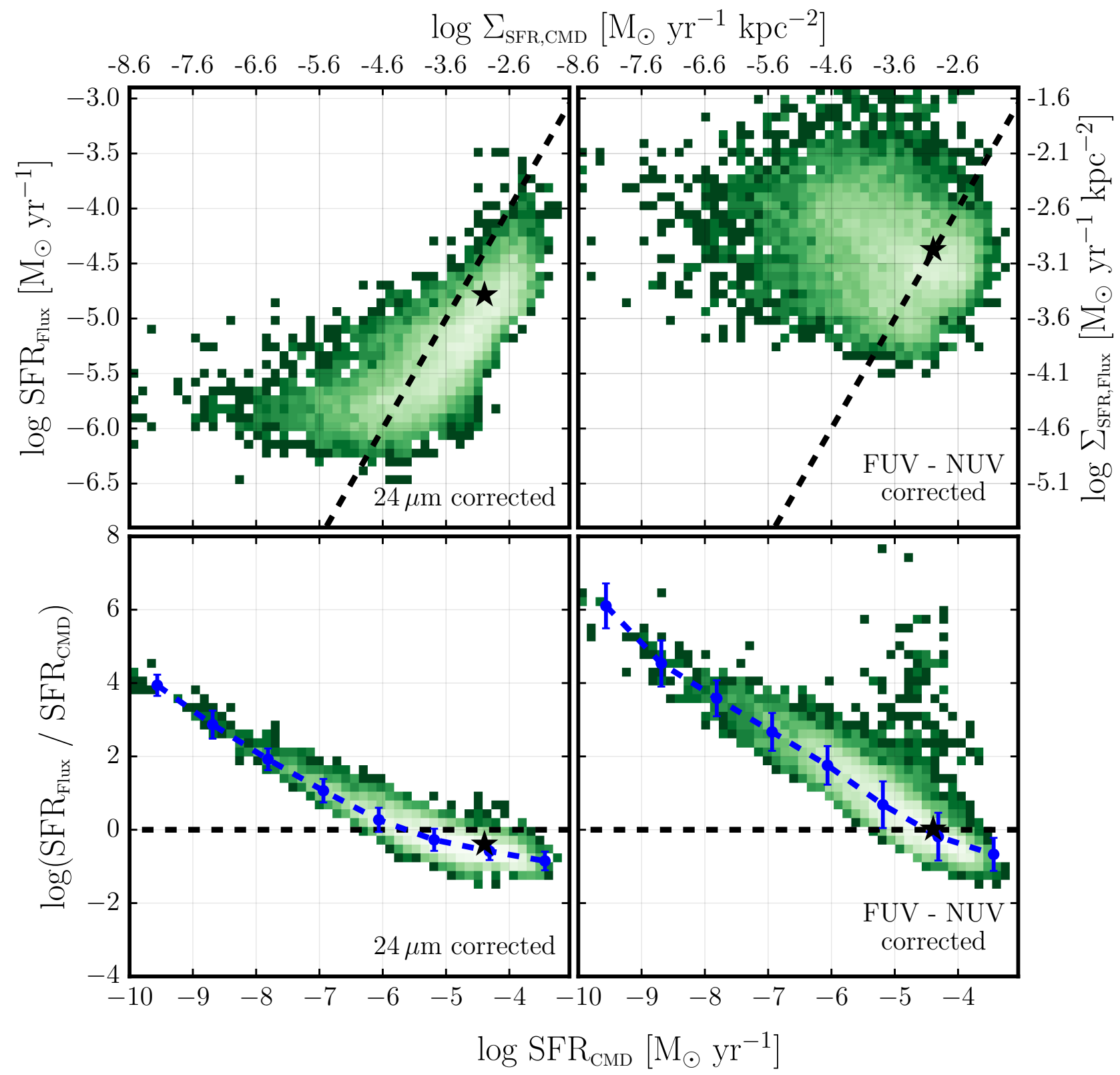

Figure 10. Comparison between CMD-based and flux-based SFRs. In all panels, the $\mathrm{x}$-axis is the SFR from the CMD-derived SFHs, averaged over the last $100 \mathrm{Myr}\left(\langle\mathrm{SFR}\rangle_{100}\right)$. In the top left panel, the y-axis shows the flux-based SFR derived from the FUV $+24 \mu \mathrm{m}$ combination. The top right panel is corrected for dust using FUV-NUV color. The bottom panels show the ratio of the flux-based SFR to the CMD-based SFR. In each panel, the black star marks the flux-weighted mean along each axis, and the black dashed line denotes one-to-one agreement.

suggests that the dust parameters derived from the SFHs are reasonable and therefore the SFR derived from the CMD-derived SFHs is not driving the discrepancy.

The inconsistencies must therefore lie with the flux calibrations. This should not be a surprising conclusion. The vast majority of SFR prescriptions are designed for regions containing at least tens of thousands of stars, such as entire galaxies or bright, star-forming regions. They make assumptions about the shape of the SFH, usually assuming a constant SFR over some timescale. They assume that the IMF in the given region is completely sampled. They assume that the region is homogenous, i.e., stellar populations are completely mixed and they are insensitive to structure in the dust, gas, and stars. Additionally, they often use only a single metallicity (usually) solar, and they are insensitive to metallicity evolution.

Most objects in the universe fall into the unresolved regime where the above assumptions are generally safe. This is especially true in the high redshift universe. In this regime, the UV color correction is commonly used to correct the SFR for dust. While Figure 10 suggests that this correction is terrible in the spatially-resolved regime, the fact that the flux-averaged mean SFRs are in agreement suggests that it may be a reasonable choice in the unresolved regime, especially when the availability of data at IR wavelengths is limited.

In the local Universe,the existing calibrations seem to 
work on large $(>1 \mathrm{kpc})$ scales, which is the scale at which they were calibrated. However, we showed in this paper that even the flux-weighted means over the entire galaxy are off when using the $24 \mu \mathrm{m}$ correction. Additionally, even on these scales, many of the above assumptions are not applicable. Nearby galaxies show highly-defined structure in spiral arms and rings, in dust lanes, and in populations of molecular clouds and other gaseous objects. This structure varies in population; spiral arms are populated by young and blue, main sequence stars. The centers of galaxies tend to contain older, more redder populations. Additionally, as structure within a galaxy is resolved, variations in stellar environment become apparent. Regions of high and low star formation, of varying metallicity, and of changing stellar density are revealed. Finally, the shape of the SFH is important, especially when correcting for dust (e.g., Boquien et al. 2016). The assumptions made when averaging over entire galaxies can no longer be made on smaller, variable scales. In fact, making such assumptions will bias the results. Nonetheless, we still want to determine SFRs for these galaxies and on these scales to take advantage of the increased spatial resolution.

In this paper, we have chosen to use a single prescription for each method of correcting FUV flux for dust. However, many studies have examined hybrid SFR tracers in an effort to determine the best combination of the data. Specifically, looking at the FUV $+24 \mu \mathrm{m}$ combination, the uncertainty primarily lies on the weight applied to the $24 \mu \mathrm{m}$ data, $w_{24}$. In this paper, we use the Hao et al. (2011) factor of 3.89, which was derived for a sample of 133 nearby, star-forming galaxies. Zhu et al. (2008) also derived a calibration between FUV and $24 \mu \mathrm{m}$ and found $w_{24}=6.31$. Both of these measurements were derived on global scales with a single data point for each galaxy.

Some work has been done on sub-galactic scales. Leroy et al. (2008) presented a calibration for the FUV + 24 $\mu \mathrm{m}$-based SFR based on a sample of 23 nearby galaxies at 750 pc scales. Modulo a typo in their appendix D, their calibration requires that $w_{24}=6.0$ (Liu et al. 2011). The Leroy et al. (2008) value of $w_{24}$ is similar to that of $\mathrm{Zhu}$ et al. (2008), with the notable difference in the physical scales on which it was derived (i.e., $750 \mathrm{pc}$ vs. entire galaxies). As discussed above, using relations on scales other than which they were derived can be precarious. In light of this, it would seem to be safer to use the Leroy et al. (2008) calibration instead of the Hao et al. (2011) calibration.

However, using $w_{24}=6.0$ does not significantly change the results presented in this study. The primary reason for this lack of change is that the calibration derived by Leroy et al. (2008) and stated in Liu et al. (2011) differs by more than just the value of $w_{24}$. The Hao et al. (2011) prescription for SFR derived from FUV + $24 \mu \mathrm{m}$ observations is:

$$
\mathrm{SFR}=4.47 \times 10^{-44}\left(L_{\mathrm{FUV}}+3.89 L_{24} \mu \mathrm{m}\right),
$$

which is simply a combination of Equations 4 and 5. The Leroy et al. (2008) prescription is:

$$
\mathrm{SFR}=3.40 \times 10^{-44}\left(L_{\mathrm{FUV}}+6.0 L_{24 \mu \mathrm{m}}\right) .
$$

The difference in the conversion factor from luminosity to SFR effectively offsets the differences that result from the change in the value of $w_{24}$. Therefore, we have chosen not to present new plots created by using this slightly different, spatially-resolved calibration factor, but instead describe the changes that result.

A change in the SFR calibration will have an effect on the fraction of obscured flux (Figure 9 and on the relationship between the flux-based SFR and the CMDbased SFR (Figure 10). In Figure 9, the primary difference is that the regions of highest flux shown in panel $\mathrm{d}$ have slightly more flux when using the Leroy et al. (2008) calibration, and the fraction of obscured star formation (panel e) is therefore higher across the galaxy. The total obscured flux increases from $72 \%$ to $80 \%$, the overall morphology of the maps does not change, and the differences discussed in Section 5.1 remain.

Increasing $w_{24}$ improves the relationship between the CMD-based SFR and the flux-based SFR improves; however, as already noted, that improvement is offset by the change in the multiplicative factor that converts a dustcorrected luminosity to a SFR. The SFR derived with the Leroy et al. (2008) prescription is offset from the CMD-based SFR by a factor of $\sim 2.3$ compared to $\sim 2.5$ for the Hao et al. (2011) calibration. Consequently, Figure 10 is largely unchanged. Therefore, while it is generally acknowledged that the global calibration of $w_{24}$ will break down on small scales, the local calibration derived on scales that are 2-8 times larger than those presented in this study - does not perform much better.

More recently, Boquien et al. (2016) calculated $w_{24}$ (they call it $k_{i}$ and examine other infrared tracers longward of $24 \mu \mathrm{m}$ as well) in 8 different galaxies and found a wide distribution ranging from 1.55 to 13.45 with a mean of 8.11. They stress that this factor has important galaxy-to-galaxy variations, as well as local vs. global variations that should not be overlooked. Uncertainty in this factor is is clearly an issue in this study as well. Had we used the Boquien et al. (2016) value of 8.11 in this paper, we would have found much better agreement between the flux-based SFR and the CMD-based SFR.

Boquien et al. (2016) actually present two different parameterizations of their factor $k_{i}$ based on FUV-NIR color and on NIR luminosity density. These parameterizations allow $k_{i}$ to vary from galaxy-to-galaxy. However, they emphasize that these parameterizations should not be used on scales below 500 pc, and therefore should not be used at the spatial scales presented here. Further testing and analysis of these parameterizations is beyond the scope of this paper. It is clear, though, that as higher spatial resolution becomes available beyond the most nearby galaxies, such methods for reliably determining the SFR while accounting for galaxy-to-galaxy variation are needed.

\section{CONCLUSIONS}

We have used spatially-resolved SFHs derived from optical resolved star data to model the SEDs of over 9000 sub-kpc regions in M31 and produce detailed maps of synthetic reddened and dust-free UV flux across the entire area covered by the PHAT survey. The SFHs were derived by Lewis et al. (2015) using F475W and F814W photometry from the PHAT survey. Both intrinsic and attenuated SEDs were derived from the SFHs using FSPS. These SEDs were convolved with the GALEX FUV and NUV response curves to generate synthetic 
fluxes, $f^{\text {syn }}$. All of the flux values were then assembled into an overall map using Montage. The pixels correspond to physical areas of $4.4 \times 10^{4} \mathrm{pc}^{2}$.

We also used Montage to construct maps of the observed UV flux, $f^{\text {obs }}$, using GALEX DIS images and 24 $\mu \mathrm{m}$ (Gordon et al. 2006), as well as $\langle\mathrm{SFR}\rangle_{100}$ from the spatially-resolved SFHs, all on the same spatial scale.

The agreement between the observed flux maps and the reddened modeled flux maps is encouraging, especially given that they were derived from only photometry in two optical bands. They indicate that models of UV emission and dust extinction are fairly accurate. The median log ratio of the synthetic reddened flux to the observed flux is 0.03 and -0.03 in the FUV and NUV, respectively, with standard deviations of 0.24 in the FUV and 0.16 in the NUV. This agreement confirms the robustness of our modeling procedure and justifies the assumptions made in the modeling routine.

The scatter in the relation between observed GALEX flux and synthetic, reddened flux is primarily due to uncertainties on the SFHs used in the modeling process. These uncertainties include a factor due to incomplete sampling of the IMF.

We compared our synthetic reddened and dust-free FUV flux maps with corresponding maps from observations. The synthetic reddened map was compared to the observed GALEX FUV map. The dust-free map was compared to map derived from a combination of FUV + $24 \mu \mathrm{m}$ data. In the synthetic FUV flux maps, $88 \%$ of the flux is obscured by dust. In the observed maps, $71 \%$ is obscured. This suggests that the $24 \mu \mathrm{m}$ correction results in an under-estimate of the total FUV flux.

We converted the observed flux map into a map of SFR using two common prescriptions, one using $24 \mu \mathrm{m}$ to correct for dust and the other using UV color. We compared the resulting SFRs with those determined from the Lewis et al. (2015) SFHs averaged over the past 100 Myr. While the morphology of the relation between the CMD-derived SFR and that calculated using the $24 \mu \mathrm{m}$ correction was good, the GALEX $+24 \mu \mathrm{m}$-derived SFR was underestimated by a factor of 2.5. Conversely, the UV-color correction resulted in a flux-weighted mean SFR in good agreement with that derived from the CMDs, but the relation shows no correlation between the two SFRs as would be expected.

The results we present in this paper provide an endto-end verification of the $\mathrm{SFH}$ results presented in Lewis et al. (2015) and show that we have the ability to model flux in a variety of bandpasses given optical data and appropriate assumptions for the stellar IMF, a set of models describing stellar spectra and evolution, and an extinction model. While modeling flux in other bands provides different challenges, the technique is extremely promising.

The authors would like to thank the anonymous referee for thoughtful comments that improved the clarity of this paper. This research has made use of NASA's Astrophysics Data System Bibliographic Services and the NASA/IPAC Extragalactic Database (NED), which is operated by the Jet Propulsion Laboratory, California Institute of Technology, under contract with the National Aeronautics and Space Administration. This work was supported by the Space Telescope Science Institute through GO-12055. This research made use of Astropy, a community-developed core Python package for Astronomy (Astropy Collaboration et al. 2013), as well as NumPy and SciPy (Oliphant 2007), IPython (Pérez \& Granger 2007), and Matplotlib (Hunter 2007). This research made use of Montage. It is funded by the National Science Foundation under Grant Number ACI-1440620, and was previously funded by the National Aeronautics and Space Administration's Earth Science Technology Office, Computation Technologies Project, under Cooperative Agreement Number NCC5-626 between NASA and the California Institute of Technology.

\section{APPENDIX}

\section{THE EFFECTS OF OLD STELLAR POPULATIONS ON SYNTHETIC AND OBSERVED FLUX}

Throughout the main body of this paper, we analyzed synthetic and observed FUV, NUV, and $24 \mu \mathrm{m}$ flux without regard to the stellar populations emitting at each wavelength. We used the entire SFH - from the present day to 14 Gyr ago - in the modeling routine (Section 3.1, despite the warning that the Lewis et al. (2015) SFHs are robust only to $500 \mathrm{Myr}$ ago). We did this in order to accurately compared the modeled UV flux with the total observed GALEX flux. While most of the UV flux comes from stars that are 100 to $300 \mathrm{Myr}$, approximately 20-30\% of the emission at FUV and NUV wavelengths comes from stars that are older than this (see discussion in Section 4.3).

We must include these older populations to accurately model the UV flux. We could have made an assumption about the form of the SFH beyond 500 Myr (constant, declining tau, etc.); however, we decided to use the full SFHs derived from the CMD analysis. We note that while the SFH solution was optimized for the most recent $500 \mathrm{Myr}$, it still has information at older ages. Using this total SFH will be more robust than using an assumed form.

\section{Modeled-to-Observed Flux Comparison}

We account for these older populations in both the synthetic and the observed data. In Figure 11, we examine the effect of older stellar populations on the synthetic flux. We plot the ratio of the synthetic flux to the observed flux as a function of the observed flux (as in Figure 4). In each panel, we have modeled the FUV flux with a different age limit applied to the SFH. The top left panel uses the full SFH and is identical to the top panel in Figure 4 . In the next panel we model the flux using the SFH back to 5 Gyr ago. The bottom left panel applies a cut to the SFH at 500 Myr ago, which is the limit specified in Lewis et al. (2015). The bottom right panel uses SFR data from just the most recent 100 Myr. In each panel, we have indicated the age limit in the top right corner. Below that is the ratio of the sum of the synthetic flux to the sum of the observed flux. The red circles and error bars show the running median and standard deviation. 


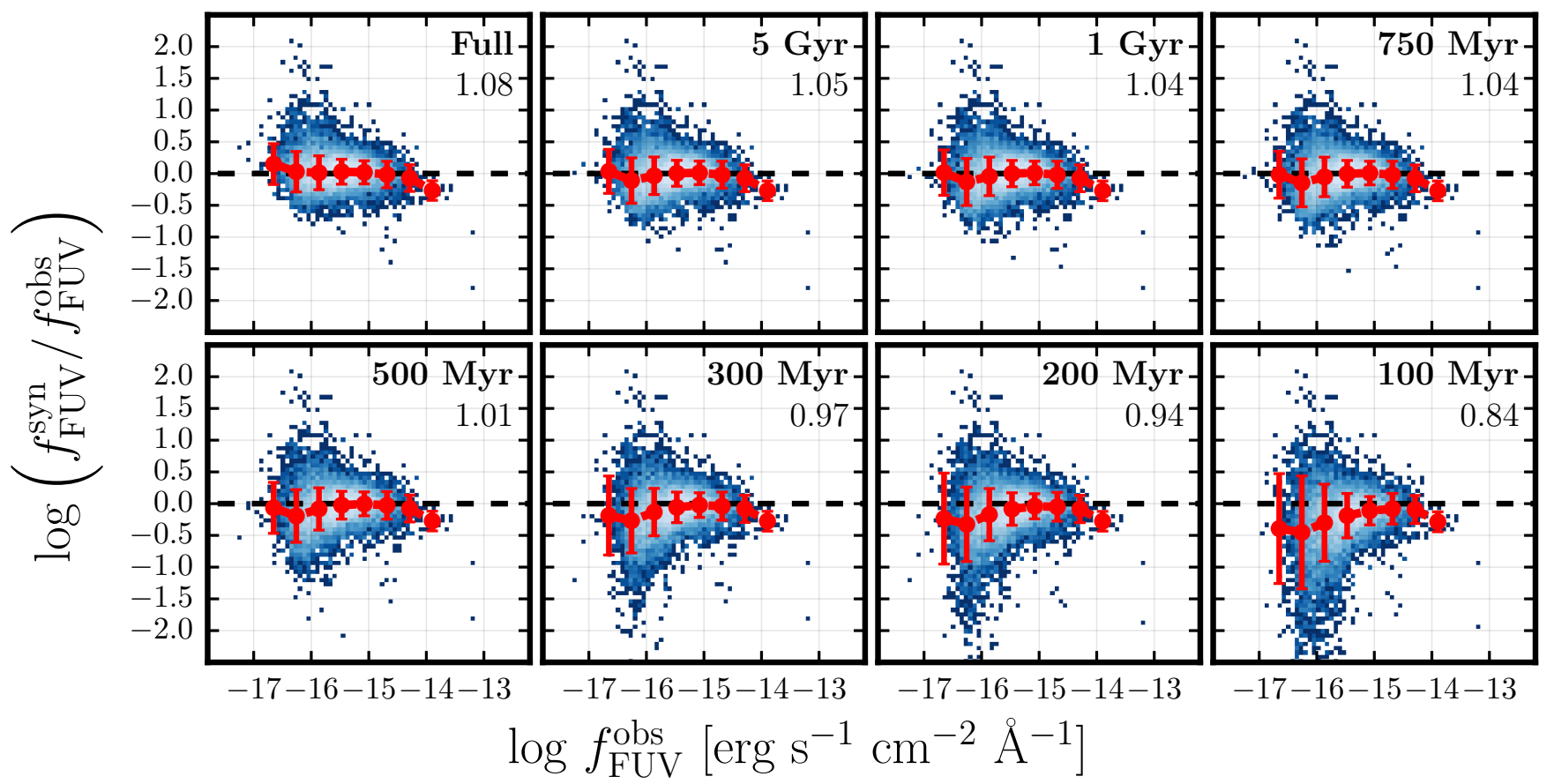

Figure 11. The log ratio of the synthetic, reddened FUV flux to the observed GALEX FUV flux as a function of observed flux with a variety of age cuts on the SFH. The red circles in each panel show the running median with the standard deviation given by the error bars on each point. The age cut used is listed in the top right corner of each panel. The fraction of total synthetic flux to total observed flux is listed just below the age cut.

The effect of reducing the age information in the flux modeling routine is clear. As the information from older populations is removed, the total synthetic flux drops, as expected. This decrease is generally seen in the low flux regions. The total synthetic flux decreases by $\sim 20 \%$ as the age information is cut from 14 Gyr to 100 yr. This is in line with literature values of the amount of UV flux expected from older populations (e.g., Johnson et al. 2013). We note, however, that in all panels and all flux bins, the ratios are consistent with zero to within $1 \sigma$.

We perform the same exercise in the NUV and plot the results in Figure 12. The general results are similar to those in the FUV except the decrease in flux is much more substantial in the NUV, especially at the low-flux end. As mentioned in our discussion of Figure 4, the NUV is much more sensitive to the shape of the extinction curve, primarily at the high-flux end. This sensitivity likely contributes to the under-production of synthetic flux seen in the top left panel when the full SFH is used and propagated through as the synthetic flux relies more strongly on younger populations that may be more affected by the $2175 \AA$ bump.

The observed data is also affected by emission from older stellar populations (e.g., Kennicutt et al. 2009). A few corrections exist in the literature. One such method is to use $3.6 \mu \mathrm{m}$ flux to correct for older populations. Some fraction of the 3.6 micron data, $\alpha_{3.6, \mathrm{FUV}}$, is subtracted from the FUV data:

$$
I_{\mathrm{FUV}, \text { young }}=I_{\mathrm{FUV}, \text { all }}-\alpha_{3.6, \mathrm{FUV}} \times I_{3.6} \mu \mathrm{m}
$$

leaving the FUV intensity from young stars. The general idea is that older stars tend to be faint at bluer wavelengths but will emit more strongly in the infrared. Leroy et al. (2008) looked at the ratio of FUV to $3.6 \mu \mathrm{m}$ SFR intensities in their sample of nearby galaxies and found $\alpha_{3.6 \text {,FUV }} \sim 2-4 \times 10^{-3}$. However, Ford et al. (2013) performed the same exercise in M31 and found $\alpha_{3.6 \text {.FUV }} \sim 8 \times 10^{-4}$. Here we use the Ford et al. (2013) value derived for M31.

We apply this correction to the observed FUV flux and we plot the resulting flux ratios in Figure 13. We have also included the same age limit cuts to the synthetic flux as we showed in Figure 11. The correction to the observed flux increases the flux ratios, especially at the low-flux end, as would be expected. As the age limit on the synthetic flux decreases, the correction to the observed flux has less of an impact. In all panels and all flux bins (except for the brightest bin), the synthetic and observed fluxes are in agreement within $1 \sigma$. There is no corresponding correction in the literature for the contribution of older populations to the NUV flux.

\section{SFR Comparison}

In this section, we examine how the effects of older stellar populations on the observed flux translate to changes in the derived SFR. In the previous section, we corrected the observed FUV flux for older stellar populations by using data at $3.6 \mu \mathrm{m}$. We can do the same thing with our $24 \mu \mathrm{m}$ data:

$$
I_{24, \text { young }}=I_{24, \text { all }}-\alpha_{3.6,24} \times I_{3.6} \mu \mathrm{m}
$$




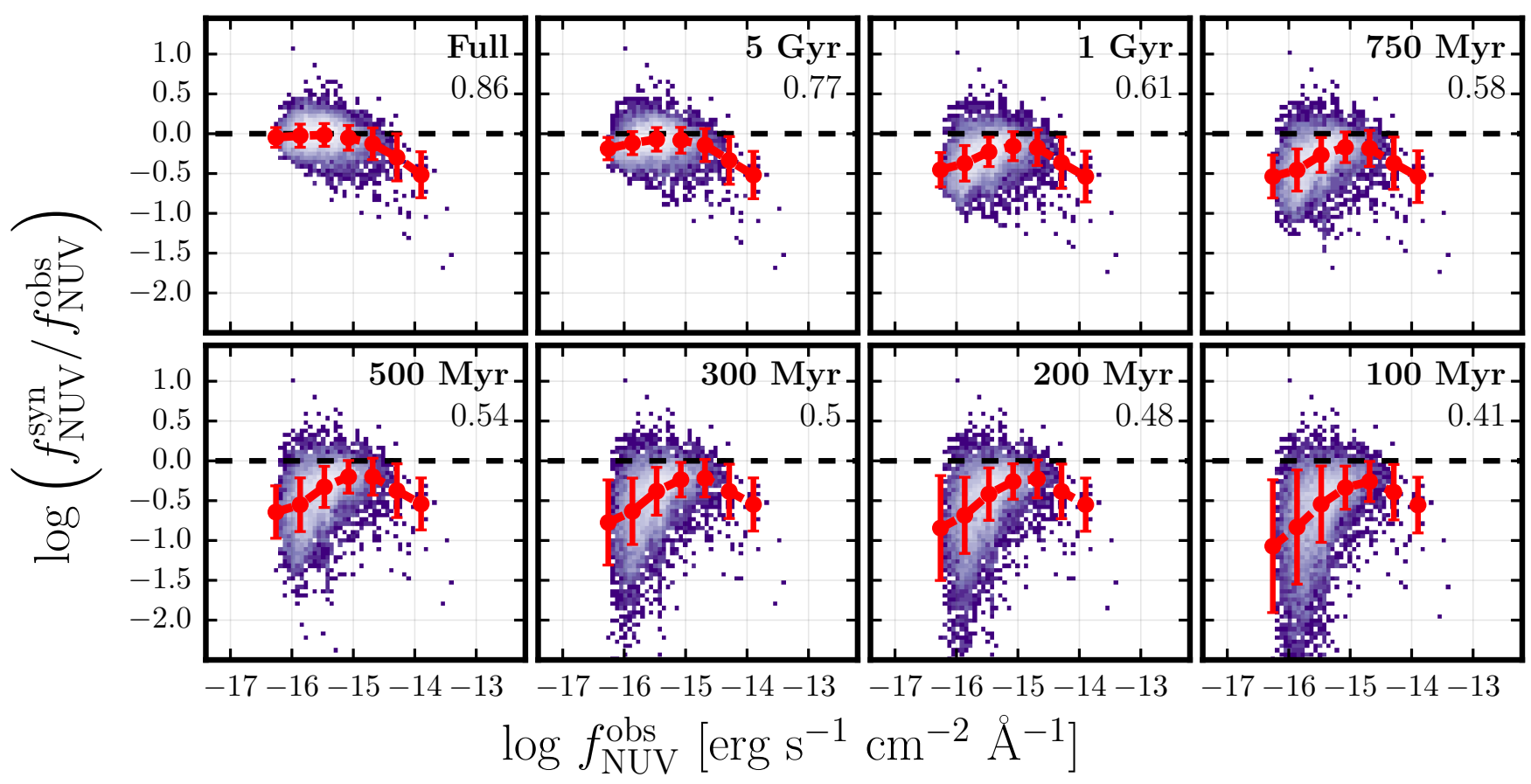

Figure 12. The same as Figure 11 except for the NUV.

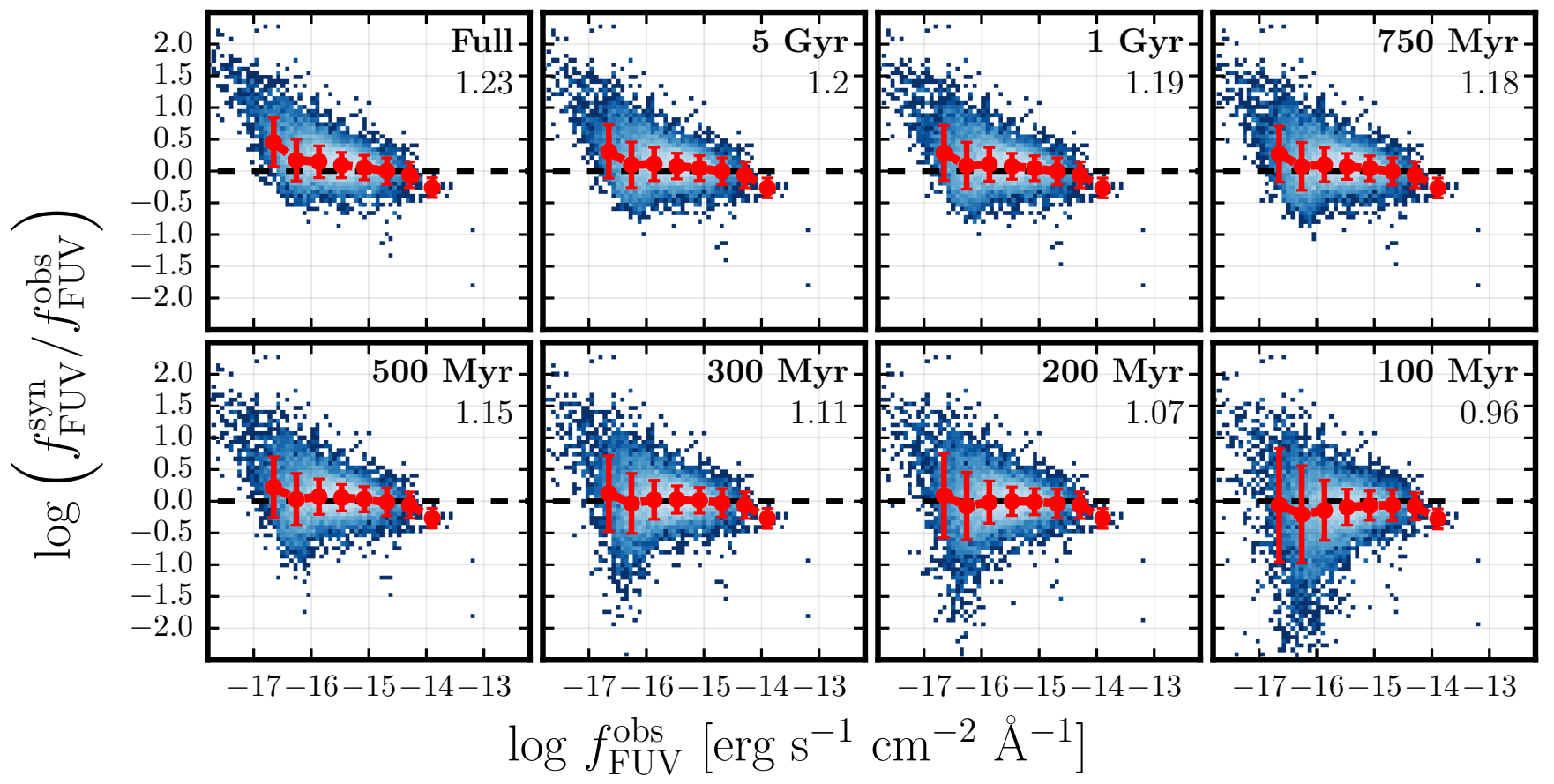

Figure 13. The same as Figure 11 except that we also include a correction for older populations in the observed flux. 
Leroy et al. (2008) found $\alpha_{3.6,24} \sim 0.1$ in their sample of galaxies. In their study of M31, Ford et al. (2013) also found $\alpha_{3.6,24}=0.1$, so we use that value here.

We corrected both the GALEX FUV and the Spitzer $24 \mu \mathrm{m}$ data for contributions from older stellar populations and recalcualted the SFRs. We plot the resulting SFRs compared with those derived from the CMD-derived SFH in Figure 14. This figure can be directly compared to Figure 10 which does not include the old star correction.

Including the correction for older stars worsens the agreement between the observed and CMD-based SFRs. In Figure 14, the x-axis remains unchanged from that in Figure 10 because it is taken directly from the most recent 100 Myr of the CMD-derived SFHs. No correction for old stars is necessary. In the left panels, the values on the y-axis decrease because there is generally less FUV flux. The overall morphology remains the same between Figures 14 and 10; however, the mean values of the flux and CMD-based SFRs now differ by 0.46 dex (a factor of 2.9), up from 0.39 dex (a factor of 2.4).

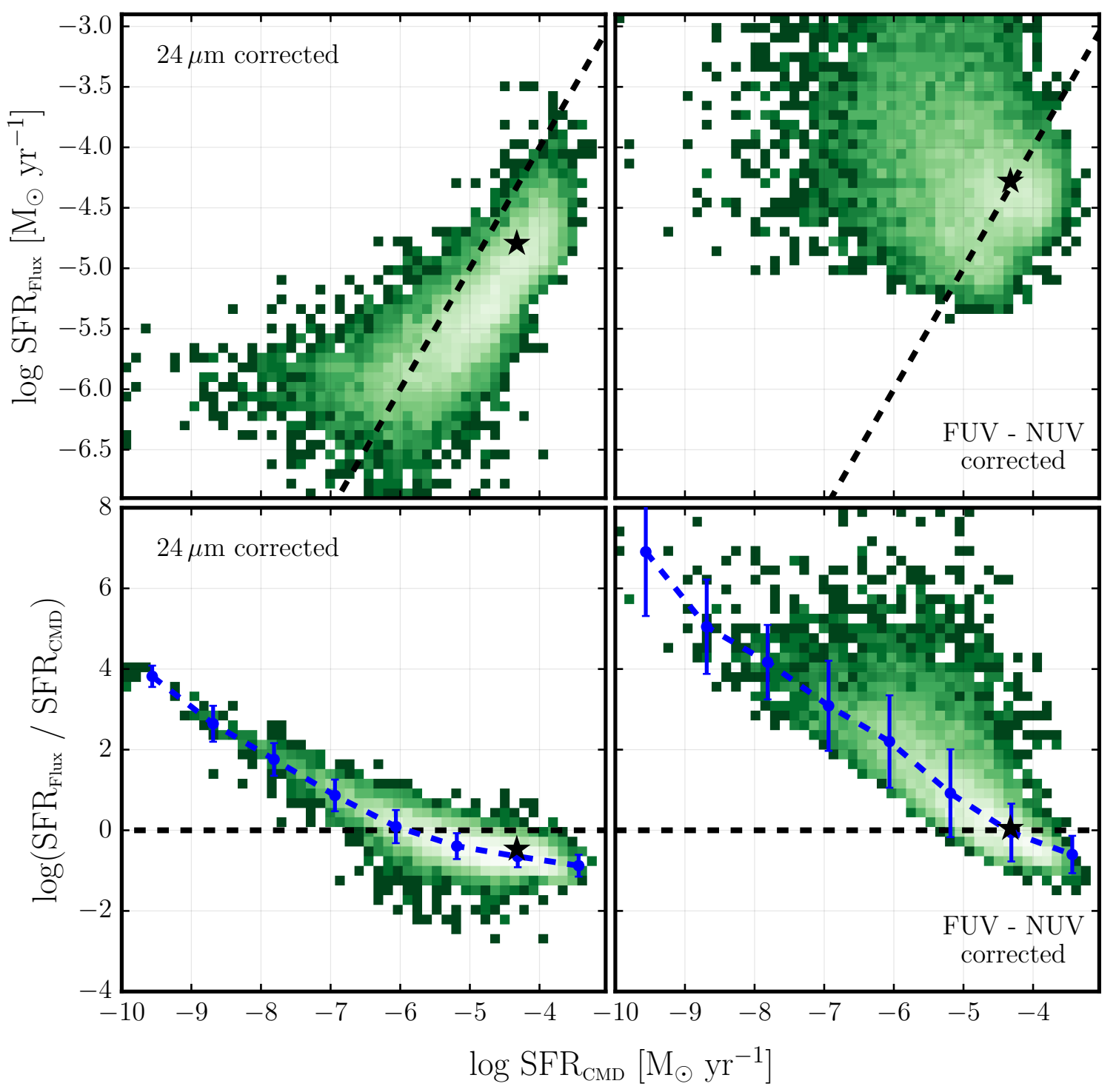

Figure 14. Comparison between CMD-based and flux-based SFRs. This is the same as Figure 10 except that we have corrected the observed FUV and $24 \mu \mathrm{m}$ fluxes for the contribution from older stellar populations. In all panels, the x-axis is the SFR from the CMDderived SFHs, averaged over the last $100 \mathrm{Myr}\left(\langle\mathrm{SFR}\rangle_{100}\right)$. In the top left panel, the y-axis shows the flux-based SFR derived from the FUV $+24 \mu \mathrm{m}$ combination. The top right panel is corrected for dust using FUV-NUV color. The bottom panels show the ratio of the flux-based SFR to the CMD-based SFR. In each panel, the black star marks the flux-weighted mean along each axis, and the black dashed line denotes one-to-one agreement.

In the right panels of Figure 14, we show the UV color-derived SFR. While the flux-weighted means along the two axes are still in agreement, there is no overall correlation between the two SFRs on small spatial scales, as seen in in Figure 10. We cannot, however, draw any conclusions from the right panels because the NUV data have not been corrected for the contribution from older stellar populations. 


\section{REFERENCES}

Astropy Collaboration, Robitaille, T. P., Tollerud, E. J., et al. 2013, A\&A, 558, A33

Bailer-Jones, C. A. L. 2011, MNRAS, 411, 435

Barmby, P., Huchra, J. P., Brodie, J. P., et al. 2000, AJ, 119, 727

Bell, E. F., \& Kennicutt, Jr., R. C. 2001, ApJ, 548, 681

Bianchi, L., Clayton, G. C., Bohlin, R. C., Hutchings, J. B., \& Massey, P. 1996, ApJ, 471, 203

Bianchi, L., Efremova, B., Hodge, P., \& Kang, Y. 2012a, AJ, 144, 142

Bianchi, L., Efremova, B., Hodge, P., Massey, P., \& Olsen, K. A. G. 2012b, AJ, 143, 74

Boquien, M., Buat, V., \& Perret, V. 2014, A\&A, 571, A72

Boquien, M., Calzetti, D., Aalto, S., et al. 2015, A\&A, 578, A8

Boquien, M., Kennicutt, R., Calzetti, D., et al. 2016, A\&A, 591, A6

Brinks, E., \& Burton, W. B. 1984, A\&A, 141, 195

Calzetti, D. 2001, PASP, 113, 1449

Calzetti, D., Kennicutt, R. C., Engelbracht, C. W., et al. 2007, ApJ, 666, 870

Cardelli, J. A., Clayton, G. C., \& Mathis, J. S. 1989, ApJ, 345, 245

Chomiuk, L., \& Povich, M. S. 2011, AJ, 142, 197

Clayton, G. C., Gordon, K. D., Bianchi, L. C., et al. 2015, ApJ, 815,14

Code, A. D. 1969, PASP, 81, 475

Conroy, C. 2013, ARA\&A, 51, 393

Conroy, C., \& Gunn, J. E. 2010, ApJ, 712, 833

Conroy, C., Gunn, J. E., \& White, M. 2009, ApJ, 699, 486

Conroy, C., Schiminovich, D., \& Blanton, M. R. 2010, ApJ, 718, 184

Crocker, A. F., Chandar, R., Calzetti, D., et al. 2015, ApJ, 808, 76

da Silva, R. L., Fumagalli, M., \& Krumholz, M. 2012, ApJ, 745, 145

da Silva, R. L., Fumagalli, M., \& Krumholz, M. R. 2014, MNRAS, 444, 3275

Dalcanton, J. J., Williams, B. F., Seth, A. C., et al. 2009, ApJS, 183, 67

Dalcanton, J. J., Williams, B. F., Lang, D., et al. 2012, ApJS, 200, 18

Dalcanton, J. J., Fouesneau, M., Hogg, D. W., et al. 2015, ApJ, 814,3

de Vaucouleurs, G., de Vaucouleurs, A., Corwin, H. G., et al. 1995, VizieR Online Data Catalog, 7155, 0

Dolphin, A. E. 2002, MNRAS, 332, 91

Dorman, B., Rood, R. T., \& O'Connell, R. W. 1993, ApJ, 419, 596

Draine, B. T., \& Li, A. 2007, ApJ, 657, 810

Duane, S., Kennedy, A., Pendleton, B. J., \& Roweth, D. 1987, Physics Letters B, 195, 216

Ford, G. P., Gear, W. K., Smith, M. W. L., et al. 2013, ApJ, 769, 55

Gehrels, N., Chincarini, G., Giommi, P., et al. 2004, ApJ, 611, 1005

Girardi, L., Williams, B. F., Gilbert, K. M., et al. 2010, ApJ, 724 1030

Gordon, K. D., Clayton, G. C., Witt, A. N., \& Misselt, K. A. 2000, ApJ, 533, 236

Gordon, K. D., Bailin, J., Engelbracht, C. W., et al. 2006, ApJL, 638, L87

Gordon, K. D., Fouesneau, M., Arab, H., et al. 2016, ApJ, 826, 104

Hao, C.-N., Kennicutt, R. C., Johnson, B. D., et al. 2011, ApJ, 741,124

Hunter, J. D. 2007, Computing in Science and Engineering, 9, 90

Johnson, B. D., Weisz, D. R., Dalcanton, J. J., et al. 2013, ApJ, 772,8
Johnson, L. C., Seth, A. C., Dalcanton, J. J., et al. 2016, ArXiv e-prints, arXiv:1606.05349

Kennicutt, R. C., \& Evans, N. J. 2012, ARA\&A, 50, 531

Kennicutt, Jr., R. C. 1998, ARA\&A, 36, 189

Kennicutt, Jr., R. C., Hao, C.-N., Calzetti, D., et al. 2009, ApJ, 703,1672

Kroupa, P. 2001, MNRAS, 322, 231

Krumholz, M. R., Fumagalli, M., da Silva, R. L., Rendahl, T., \& Parra, J. 2015, MNRAS, 452, 1447

Lee, J. C., Gil de Paz, A., Tremonti, C., et al. 2009, ApJ, 706, 599

Lee, J. C., Gil de Paz, A., Kennicutt, Jr., R. C., et al. 2011, ApJS, 192, 6

Leroy, A. K., Walter, F., Brinks, E., et al. 2008, AJ, 136, 2782

Leroy, A. K., Bigiel, F., de Blok, W. J. G., et al. 2012, AJ, 144, 3

Lewis, A. R., Dolphin, A. E., Dalcanton, J. J., et al. 2015, ApJ, 805,183

Liu, G., Koda, J., Calzetti, D., Fukuhara, M., \& Momose, R. 2011, ApJ, 735, 63

Maíz-Apellániz, J. 2004, PASP, 116, 859

Marcum, P. M., O'Connell, R. W., Fanelli, M. N., et al. 2001, ApJS, 132,129

Marigo, P., Girardi, L., Bressan, A., et al. 2008, A\&A, 482, 883

Martin, D. C., Fanson, J., Schiminovich, D., et al. 2005, ApJL, 619, L1

McConnachie, A. W., Irwin, M. J., Ferguson, A. M. N., et al 2005, MNRAS, 356, 979

Meurer, G. R., Heckman, T. M., \& Calzetti, D. 1999, ApJ, 521, 64

Meurer, G. R., Wong, O. I., Kim, J. H., et al. 2009, ApJ, 695, 765

Meynet, G., Maeder, A., Schaller, G., Schaerer, D., \& Charbonnel, C. 1994, A\&AS, 103

Morrissey, P., Conrow, T., Barlow, T. A., et al. 2007, ApJS, 173, 682

Moustakas, J., \& Kennicutt, Jr., R. C. 2006, ApJS, 164, 81

Murphy, E. J., Condon, J. J., Schinnerer, E., et al. 2011, ApJ, 737,67

O'Connell, R. W. 1999, ARA\&A, 37, 603

O'Connell, R. W., Bohlin, R. C., Collins, N. R., et al. 1992 , ApJL, 395, L45

Oliphant, T. E. 2007, Computing in Science \& Engineering, 9, 10

Pérez, F., \& Granger, B. 2007, Computing in Science

Engineering, 9, 21

Pradhan, A. C., Ojha, D. K., Robin, A. C., Ghosh, S. K., \& Vickers, J. J. 2014, A\&A, 565, A33

Roberts, M. S. 1966, ApJ, 144, 639

Robitaille, T. P., Whitney, B. A., Indebetouw, R., \& Wood, K. 2007, ApJS, 169, 328

Romaniello, M., Panagia, N., Scuderi, S., \& Kirshner, R. P. 2002, AJ, 123,915

Rosenfield, P., Johnson, L. C., Girardi, L., et al. 2012, ApJ, 755, 131

Schmitt, H. R., Calzetti, D., Armus, L., et al. 2006, ApJ, 643, 173

Simones, J. E., Weisz, D. R., Skillman, E. D., et al. 2014, ApJ, 788,12

Thilker, D. A., Hoopes, C. G., Bianchi, L., et al. 2005, ApJL, 619, L67

van den Bergh, S. 1964, ApJS, 9, 65

Walterbos, R. A. M., \& Kennicutt, Jr., R. C. 1988, A\&A, 198, 61

Weisz, D. R., Dalcanton, J. J., Williams, B. F., et al. 2011, ApJ, 739,5

Weisz, D. R., Johnson, B. D., Johnson, L. C., et al. 2012, ApJ, 744,44

Westera, P., Lejeune, T., Buser, R., Cuisinier, F., \& Bruzual, G. 2002, A\&A, 381, 524

Wilkins, S. M., Gonzalez-Perez, V., Lacey, C. G., \& Baugh, C. M. 2012, MNRAS, 427, 1490

Williams, B. F., Lang, D., Dalcanton, J. J., et al. 2014, ApJS, 215, 9

Zhu, Y.-N., Wu, H., Cao, C., \& Li, H.-N. 2008, ApJ, 686, 155 